\title{
Study of nucleon resonances with electromagnetic interactions
}

\author{
(From EBAC, Thomas Jefferson National Accelerator \\ Facility)
}

\author{
T.-S. H. Lee ${ }^{1}$, L.C. Smith ${ }^{2}$ \\ ${ }^{1}$ Physics Division, Argonne National Laboratory, Argonne, Illinois 60439, USA \\ and \\ Excited Baryon Analysis Center, Thomas Jefferson National Accelerator \\ Facility, Newport News, Virginia 23606, USA \\ E-mail: lee@phy.anl.gov \\ ${ }^{2}$ Physics Department, University of Virginia, Charlottesville, Virginia 22901, \\ USA
}

\begin{abstract}
Recent developments in using electromagnetic meson production reactions to study the structure of nucleon resonances are reviewed. Possible future works are discussed.
\end{abstract}

\section{Introduction}

The study of nucleon resonances $\left(N^{*}\right)$ has long been recognized as an essential step towards developing a fundamental understanding of strong interactions. Since the discovery of the $\Delta(1232)$ in 1951 , many $N^{*}$ 's have been identified mainly through amplitude analyses of pion-nucleon elastic scattering. This effort peaked in the 1970's with fairly consistent results from several independent groups. A longstanding challenge in hadronic physics is to understand the spectroscopy of these resonant states, including their electromagnetic and strong decays, within a framework consistent with QCD. However, many of the assumptions used in phenomenological analyses remain to be justified theoretically. Also, the limitations of the data base lead to rather large errors in many of the determined resonance parameters.

Some additional but limited pion-nucleon scattering data have been obtained since the 1980's. While these data have improved the determination of $\pi N$ amplitudes at low energies, there has not been much impact in the $N^{*}$ region. The situation changed drastically in the 1990's with the construction of new electron and photon beam facilities. Experiments at Jefferson Laboratory, MIT-Bates, LEGS, Mainz, Bonn, GRAAL, and Spring- 8 are providing new data on the photo- and electroproduction of $\pi, \eta, K, \omega, \phi$ mesons and $2 \pi$ final states. These data offer a new opportunity to understand in detail how $N^{*}$ properties emerge from the non-perturbative aspects of

$\ddagger$ Notice: Authored by Jeffers on Science Associates, LLC under U.S. DOE Contract No. DE-AC0506OR23177. The U.S. Government retains a non-exclusive, paid-up, irrevocable, world-wide license to publish or reproduce this manuscript for U.S. Government purposes. 
QCD. For example, the extraction of $\gamma N \rightarrow N^{*}$ transition form factors could shed light on the dynamical origins of the confinement of constituent quarks and the associated meson cloud.

To make progress, we need to perform amplitude analyses of the data to extract the $N^{*}$ parameters. More importantly, we need to develop dynamical reaction models to interpret the extracted $N^{*}$ parameters in terms of QCD. At the present time, the achievable goal is to test the predictions from various QCD-based hadron models such as the well-developed constituent quark model[1] and the covariant model based on Dyson-Schwinger Equations [2]. In the near future, we hope to understand the $N^{*}$ parameters in terms of Lattice QCD (LQCD).

In the $\Delta$ region, both the amplitude analyses and reaction models have been well developed. We find that these two efforts are complementary. For example, a recent review of amplitude analyses [3] gave the result that the $N-\Delta \mathrm{M} 1$ transition strength is $G_{M}(0)=3.18 \pm 0.04$. This value is about $40 \%$ larger than the constituent quark model predictions $G_{M}(0)=\frac{4}{3} \sqrt{\frac{m_{N}}{2 m_{\Delta}}} G_{p}(0) \sim 2.30$. Using dynamical models [4, 5] one can show that the discrepancy is due to the pion cloud which is not included in the constituent quark model calculations. The effect of the meson cloud on the $N-\Delta$ transition has been further revealed in the study of pion electroproduction reactions.

In the higher mass $N^{*}$ region, the situation is much more complicated because of many open channels. Any reliable analysis of the meson production data in this higher energy region must be based on a coupled-channel approach. In recent years, significant progress has been made in this direction.

In this article, we will briefly summarize the status of theoretical and experimental efforts in the study of $N^{*}$ resonances. In section 2, we review most of the current meson production reaction models used to extract $N^{*}$ information from meson production data. In section 3, we summarize the results from recent analyses of photo- and electro-production data. The focus will be on the $N-\Delta$ (1232) transition form factors which have now been investigated in great detail. We will also summarize the $N-N^{*}$ transition form factors extracted recently by several groups. Discussions on future developments will be given in section 4 .

\section{Theoretical developments}

Most of the models for meson production reactions can be derived by considering the following coupled-channel equations

$$
T_{a, b}(E)=V_{a, b}+\sum_{c} V_{a, c} g_{c}(E) T_{c, b}(E),
$$

where $a, b, c=\gamma N, \pi N, \eta N, \omega N, K Y, \pi \Delta, \rho N \sigma N, \cdots$. The interaction term is defined by $V_{a, b}=<a|V| b>$ with

$$
V=v^{b g}+v^{R} .
$$

Here $v^{b g}$ represents the non-resonant(background) mechanisms such as the tree diagrams illustrated in Figs. 1(a)-(d), and $v^{R}$ describes the $N^{*}$ excitation shown in Figure1(e). Schematically, the resonant term can be written as

$$
v^{R}(E)=\sum_{N_{i}^{*}} \frac{\Gamma_{i}^{\dagger} \Gamma_{i}}{E-M_{i}^{0}},
$$




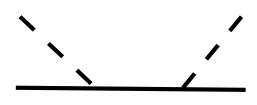

(a)

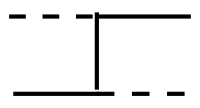

(b)

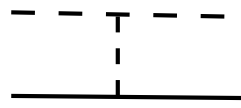

(c)

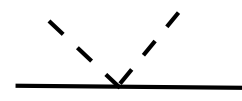

(d)

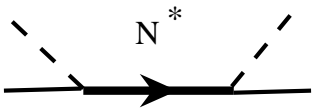

(e)

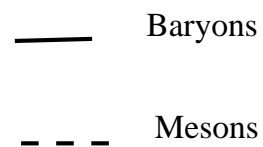

Figure 1. Tree-diagram mechanisms of meson-baryon interactions.

where $\Gamma_{i}$ defines the decay of the $i$-th $N^{*}$ state into meson-baryon states, and $M_{i}^{0}$ is a mass parameter related to the resonance position.

The meson-baryon propagator in (11) is

$$
g_{c}(E)=<c|g(E)| c>,
$$

with

$$
\begin{aligned}
g(E) & =\frac{1}{E-H_{0}+i \epsilon} \\
& =g^{P}(E)-i \pi \delta\left(E-H_{0}\right),
\end{aligned}
$$

where $H_{0}$ is the free Hamiltonian and

$$
g^{P}(E)=\frac{P}{E-H_{0}} .
$$

Here $P$ denotes taking the principal-value part of any integration over the propagator. If $g(E)$ is replaced by $g^{P}(E)$ and $T_{a, b}(E)$ by $K_{a, b}(E)$, (1) then defines the K-matrix which is related to T-matrix by

$$
T_{a, b}(E)=K_{a, b}(E)-\sum_{c} T_{a, c}(E)\left[i \pi \delta\left(E-H_{0}\right)\right]_{c} K_{c, b}(E) .
$$

By using the two potential formulation, one can cast (1) into

$$
T_{a, b}(E)=t_{a, b}^{b g}(E)+t_{a, b}^{R}(E)
$$

with

$$
t_{a, b}^{R}(E)=\sum_{N_{i}^{*}, N_{j}^{*}} \bar{\Gamma}_{N_{i}^{*}, a}^{\dagger}(E)[G(E)]_{i, j} \bar{\Gamma}_{N_{j}^{*}, b}(E) .
$$

The first term of (17) is determined only by the non-resonant interaction

$$
t_{a, b}^{b g}(E)=v_{a, b}^{b g}+\sum_{c} v_{a, c}^{b g} g_{c}(E) t_{c, b}^{b g}(E) .
$$

The resonant amplitude (8) is determined by the dressed vertex

$$
\bar{\Gamma}_{N^{*}, a}(E)=\Gamma_{N^{*}, a}+\sum_{b} \Gamma_{N^{*}, b} g_{b}(E) t_{b, a}^{b g}(E),
$$

and the dressed propagator

$$
\left[G(E)^{-1}\right]_{i, j}(E)=\left(E-M_{i}^{0}\right) \delta_{i, j}-\Sigma_{i, j}(E) .
$$


Here the mass-shift term is

$$
\Sigma_{i, j}(E)=\sum_{a} \Gamma_{N^{*}, a}^{\dagger} g_{a}(E) \bar{\Gamma}_{N_{j}^{*}, a}(E) .
$$

Note that the meson-baryon propagator $g_{a}(E)$ for channels including an unstable particle, such as $\pi \Delta, \rho N$ and $\sigma N$, must be modified to include a width.

With the above equations, we now can derive in the next few subsections most of the recent models for analyzing the data of meson production reactions.

\subsection{Unitary Isobar Model}

In recent years, the most widely used model is the Unitary Isobar Model (UIM) developed [6] by the Mainz group. This model, called MAID, is based on the onshell relation (6). By including only one hadronic channel, $\pi N$ (or $\eta N$ ), (6) leads to

$$
T_{\pi N, \gamma N}=e^{i \delta_{\pi N}} \cos \delta_{\pi N} K_{\pi N, \gamma N},
$$

where $\delta_{\pi N}$ is the pion-nucleon scattering phase shift. By further assuming that $K \rightarrow V=v^{b g}+v^{R}$, (13) can be cast into the following form

$$
T_{\pi N, \gamma N}(U I M)=e^{\delta_{\pi N}} \cos \delta_{\pi N}\left[v_{\pi N, \gamma N}^{b g}\right]+\sum_{N_{i}^{*}} T_{\pi N, \gamma N}^{N_{i}^{*}}(E) .
$$

The non-resonant term $v^{b g}$ in (14) is calculated from the standard Born terms but with an energy-dependent mixture of pseudo-vector (PV) and pseudo-scalar (PS) $\pi N N$ coupling. For the resonant term in (14), MAID uses the following parameterization by Walker [7]

$$
T_{\pi N, \gamma N}^{N_{i}^{*}}(E)=f_{\pi N}^{i}(E) \frac{\Gamma_{t o t} M_{i} e^{i \Phi}}{M_{i}^{2}-E^{2}-i M_{i} \Gamma^{t o t}} f_{\gamma N}^{i}(E) \bar{A}^{i},
$$

where $f_{\pi N}^{i}(E)$ and $f_{\gamma N}^{i}(E)$ are the form factors describing the decays of $N^{*}, \Gamma^{t o t}$ is the total decay width, and $\bar{A}^{i}$ is the $\gamma N \rightarrow N^{*}$ excitation strength. The phase $\Phi$ is required by the unitarity condition and is determined by an assumption that relates the phase of the total photoproduction amplitude to the $\pi N$ scattering phase shift.

The UIM developed by the JLab-Yerevan collaboration [8, 9, 10] is similar to MAID. The main difference is that this model uses the Regge parameterization to define the amplitudes at high energies. Schematically, they write non-resonant amplitude [Background $]=\left[e^{\delta_{\pi N}} \cos \delta_{\pi N} v^{b g}\right]$ of (14) as

$$
\begin{aligned}
{[\text { Background }]=} & {[\text { Born }+\rho+\omega]_{U I M} \text { at } s<s_{0} } \\
= & {[\text { Born }+\rho+\omega]_{U I M} \frac{1}{1+\left(s-s_{0}\right)^{2}} } \\
& +[\text { Regge }- \text { Poles }] \frac{\left(s-s_{0}\right)^{2}}{1+\left(s-s_{0}\right)^{2}} \text { at } s>s_{0}
\end{aligned}
$$

where $s_{0} \sim 1.2 \mathrm{GeV}$ was determined phenomenologically in fitting the pion photoproduction data.

Both MAID and JLab-Yeveran UIM have been applied extensively to analyze the data of $\pi$ and $\eta$ production reactions. 


\subsection{K-matrix Coupled-Channel Models}

2.2.1. VPI-GWU Model The VPI-GWU [1] (SAID) can be derived from (6) by considering three channels: $\gamma N, \pi N$, and $\pi \Delta$. The solution of the resulting $3 \times 3$ matrix equation leads to

$$
T_{\gamma N, \pi N}(S A I D)=A_{I}\left(1+i T_{\pi N, \pi N}\right)+A_{R} T_{\pi N, \pi N},
$$

where

$$
\begin{aligned}
& A_{I}=K_{\gamma N, \pi N}-\frac{K_{\gamma N, \pi \Delta} K_{\pi N, \pi N}}{K_{\pi N, \pi \Delta}}, \\
& A_{R}=\frac{K_{\gamma N, \pi \Delta}}{K_{\pi N, \pi \Delta}} .
\end{aligned}
$$

The $\pi N$ amplitude $T_{\pi N, \pi N}$ needed to evaluate (18) is available in SAID. In actual analyses, they simply parameterize $A_{I}$ and $A_{R}$ as polynomial functions of the on-shell momenta for pion and photon with adjustable parameters. Once the parameters of $A_{I}$ and $A_{R}$ have been determined by fitting the pion photoproduction data, the $N^{*}$ parameters are extracted by fitting the resulting amplitude $T_{\gamma N, \pi N}$ at energies near the resonance position to a Breit-Wigner parameterization (similar to (15)).

An extensive database of pion photoproduction experiments has been analyzed by SAID. The extension of SAID to the analysis of pion electroproduction data is being pursued.

2.2.2. Giessen and KVI Models The K-matrix coupled-channel models developed by the Giessen group [12, 13, 14, 15] and the KVI group [16] can be obtained from (6) by taking the approximation $K=V$. This leads to a matrix equation involving only the on-shell matrix elements of $V$

$$
T_{a, b}(E) \rightarrow \sum_{c}\left[(1+i V(E))^{-1}\right]_{a, c} V_{c, b}(E) .
$$

The interaction $V=v^{b g}+v^{R}$ is calculated from tree diagrams such as those illustrated in Figure 1. The Giessen group has performed analyses [12, 13, 14, 15] with $\gamma N, \pi N$, $2 \pi N, \eta N, \omega N$, and $K \Lambda$ channels. They further simplify $2 \pi N$ as a stable partice channel. The KVI group [16] has focused on the hyperon production reactions by performing analyses with $\gamma N, \pi N, \eta N, K \Lambda, K \Sigma, \phi N$ channels.

\subsection{Dynamical Models}

The dynamical models of meson-baryon reactions account for the off-shell scattering dynamics through the use of integral equations such as (11) or their equivalence (77)-(12). The off-shell dynamics are closely related to the meson-baryon scattering wavefunctions in the short-range region where we want to map out the structure of $N^{*}$. Thus the development of dynamical models is an important step towards interpreting the extracted $N^{*}$ parameters.

In recent years, the predictions from the dynamical models of Sato and Lee (SL) [4] and the Dubna-Mainz-Taiwan (DMT) collaboration [5] are most often used to analyze the data in the $\Delta$ region. The SL model can be derived from (77)-(12) by keeping only one resonance $N^{*}=\Delta$ and two channels $a, b=\pi N, \gamma N$. In solving exactly the

resulting equations the non-resonant interactions $v_{\pi N, \pi N}^{b g}$ and $v_{\pi N, \gamma N}^{b g}$ are derived from the standard PV Born terms and $\rho$ and $\omega$ exchanges by using an unitary transformation method. 
The DMT model also includes only $\pi N$ and $\gamma N$ channels. They however depart from the exact formulation based on (11) or (7)-(12) by using the Walker parameterization (15) to describe the resonant amplitude. Accordingly, their definition of the non-resonant amplitude also differs from that defined by (9) $: t_{c, b}^{b g}$ in the righthand side of (9) is replaced by the full amplitude $T_{c, b}$. Furthermore, they follow MAID to calculate the non-resonant interaction $v_{\pi N, \gamma N}^{b g}$ from an energy-dependent mixture of PS and PV Born terms.

Extensive data of pion photoproduction and electroproduction in the $\Delta$ region can be described by both the SL and DMT models. However, they have significant differences in the extracted electric E2 and Coulomb C2 form factors of the $\gamma N \rightarrow \Delta$ transition. Both models show very large pion cloud effects on the $\gamma N \rightarrow \Delta$ transition form factors in the low $Q^{2}$ region.

The Utrecht-Ohio model [17] has also succeeded in describing the data in the $\Delta$ region. Despite some differences in treating the gauge invariance problem and the $\Delta$ excitation amplitude, its dynamical content is similar to that of SL and DMT models.

The equations (7)-(12) are used in a $2-N^{*}$ and 3 -channels $(\pi N, \eta N$, and $\pi \Delta)$ study [18, of $\pi N$ scattering in $S_{11}$ partial wave. This work illustrated the extent to which the quark-quark interactions in the constituent quark model can be determined directly by the $\pi N$ reaction data. The equations (7)-(12) have also been used to show that the coupled-channel effects due to the $\pi N$ channel are very large in $\omega$ photoproduction [19] and $K$ photoproduction [20. All $N^{*}$ identified by the Particle Data Group are included in these two dynamical calculations.

The coupled-channel study of both $\pi N$ scattering and $\gamma N \rightarrow \pi N$ in the $S_{11}$ channel by Chen et al 21] includes $\pi N, \eta N$, and $\gamma N$ channels. Their $\pi N$ scattering calculation is performed by using (1). For their $\gamma N \rightarrow \pi N$ calculation, they neglect the $\gamma N \rightarrow \eta N \rightarrow \pi N$ channel coupling, and follow the procedure of the DMT model to evaluate the resonant term in terms of the Walker parameterization (15). They find that four $N^{*}$ are needed to fit the empirical amplitudes in the $S_{11}$ channel up to $W=2 \mathrm{GeV}$. This approach is being extended to also fit the $\pi N \rightarrow \pi N$ and $\gamma N \rightarrow \pi N$ amplitudes in all partial waves.

A coupled-channel calculation based on (10) has been carried out by the Jülich group 22 for $\pi N$ scattering. They are able to describe the $\pi N$ phase shifts up to $W=1.9 \mathrm{GeV}$ by including the $\pi N, \eta N, \pi \Delta, \rho N$ and $\sigma N$ channels and $5 N^{*}$ resonances: $P_{33}(1232), S_{11}(1535), S_{11}(1530), S_{13}(1650)$ and $D_{13}(1520)$. They find that the Roper resonance $P_{11}(1440)$ arises completely from the meson-exchange coupledchannel effects.

A coupled channel calculation based on (11) for both the $\pi N$ scattering and $\gamma N \rightarrow \pi N$ up to $W=1.5 \mathrm{GeV}$ has been reported by Fuda and Alarbi [23]. They include the $\pi N, \gamma N, \eta N$ and $\pi \Delta$ channels and $4 N^{*}$ resonances: $P_{33}(1232), P_{11}(1440)$, $S_{11}(1535)$ and $D_{13}(1520)$. They adjust the parameters of their model to fit the empirical multipole amplitudes in low partial waves.

More simple coupled-channel calculations have been performed by using separable interactions. In the model of Gross and Surya 24, separable interactions come from simplifying the meson-exchange mechanisms in Figs 1(a)-1(c) as a contact term like Figure 1(d). They include only $\pi N$ and $\gamma N$ channels and 3 resonances: $P_{33}(1232)$, $P_{11}(1440)$ and $D_{13}(1520)$, and restrict their investigation up to $W<1.5 \mathrm{GeV}$. To account for the inelasticities in $P_{11}$ and $D_{13}$, the $N^{*} \rightarrow \pi \Delta$ coupling is introduced in these two partial waves. The inelasticities in other partial waves are neglected. 
A similar separable simplification is also used in the chiral coupled-channel models 25, 26, for strange particle production. There the separable interactions are directly deduced from the $\mathrm{SU}(3)$ effective chiral lagrangians. They are able to fit the total cross section data for various strange particle production reaction channels without introducing $N^{*}$ resonance states. In recent years, this model has been further extended by Lutz and Kolomeitsev [27] to also fit the $\pi N$ scattering data.

None of the dynamical models described above account for all of the effects arising from the $\pi \pi N$ channels, which contribute about $1 / 2$ of the $\pi N$ and $\gamma N$ total cross sections in the higher mass $N^{*}$ region. Consequently, the $N^{*}$ parameters extracted from these models could have uncertainties due to the violation of the $\pi \pi N$ unitarity condition. One straightforward way to improve the situation is to extend the Hamiltonian formulation of [4] to include (a) $\rho \rightarrow \pi \pi$ and $\sigma \rightarrow \pi \pi$ decay mechanisms as specified in $\Gamma_{V}$ of (24), (b) $v_{\pi \pi}$ for non-resonant $\pi \pi$ interactions, (c) $v_{M N, \pi \pi N}$ for non-resonant $M N \rightarrow \pi \pi N$ transitions with $M N=\gamma N$ or $\pi N$, and (d) $v_{\pi \pi N, \pi \pi N}$ for non-resonant $\pi \pi N \rightarrow \pi \pi N$ interactions. Such a dynamical coupled-channel model has been developed recently by Matsuyama, Sato, and Lee 28 (MSL). The coupledchannel equations from this model can also be cast into the form of (7)-(12) except that the driving term of (9) is replaced by

$$
v_{a, b}^{b g} \rightarrow \hat{V}_{a, b}=v_{a, b}^{b g}+Z_{a, b}(E),
$$

where

$$
\begin{aligned}
Z_{a, b}(E)= & <a\left|F \frac{P_{\pi \pi N}}{E-H_{0}-v_{\pi N, \pi N}-v_{\pi \pi}-v_{\pi \pi N, \pi \pi N}+i \epsilon} F^{\dagger}\right| b> \\
& -<a\left|\Sigma_{V}(E)\right| b>\delta_{a, b}
\end{aligned}
$$

with

$$
\begin{aligned}
F & =\Gamma_{V}+v_{\pi N, \pi \pi N} \\
& =\Gamma_{\Delta, \pi N}+h_{\rho, \pi \pi}+h_{\sigma, \pi \pi}+v_{\pi N, \pi \pi N} .
\end{aligned}
$$

Note that $\Sigma_{V}(E)$ in (23) describes the self-energy of unstable particle channels $\pi \Delta, \rho N$, and $\sigma N$. Obviously $Z_{a, b}(E)$ contains the non-resonant multiple scattering mechanisms within the $\pi \pi N$ subspace. It generates $\pi \pi N$ unitarity cuts. It has been shown in 28 that this term has very large effects on $2 \pi$ production reactions. This dynamical coupled-channel model is currently being further developed at the Excited Baryon Analysis Center (EBAC) at JLab.

\section{Experimental developments}

The past ten years has seen an unprecedented increase in the volume and precision of $N^{*}$ data from electromagnetic meson production reactions. This has resulted from the development of $\mathrm{CW}$ electron accelerators, which drastically reduce accidental backgrounds in coincidence experiments, as well as from advances in charged, neutron and photon detector technologies such as nearly $4 \pi$ solid angle coverage and improved spatial/energy resolution. In addition, the development of polarized beams, targets and polarimeters has opened up a previously unavailable set of observables which are sensitive to the interference between resonant and non-resonant processes in the scattering amplitude. An extensive survey of initial data from the new facilities can be found in the review by Burkert and Lee [31. In this section we highlight results from recent analyses of ongoing experiments. 

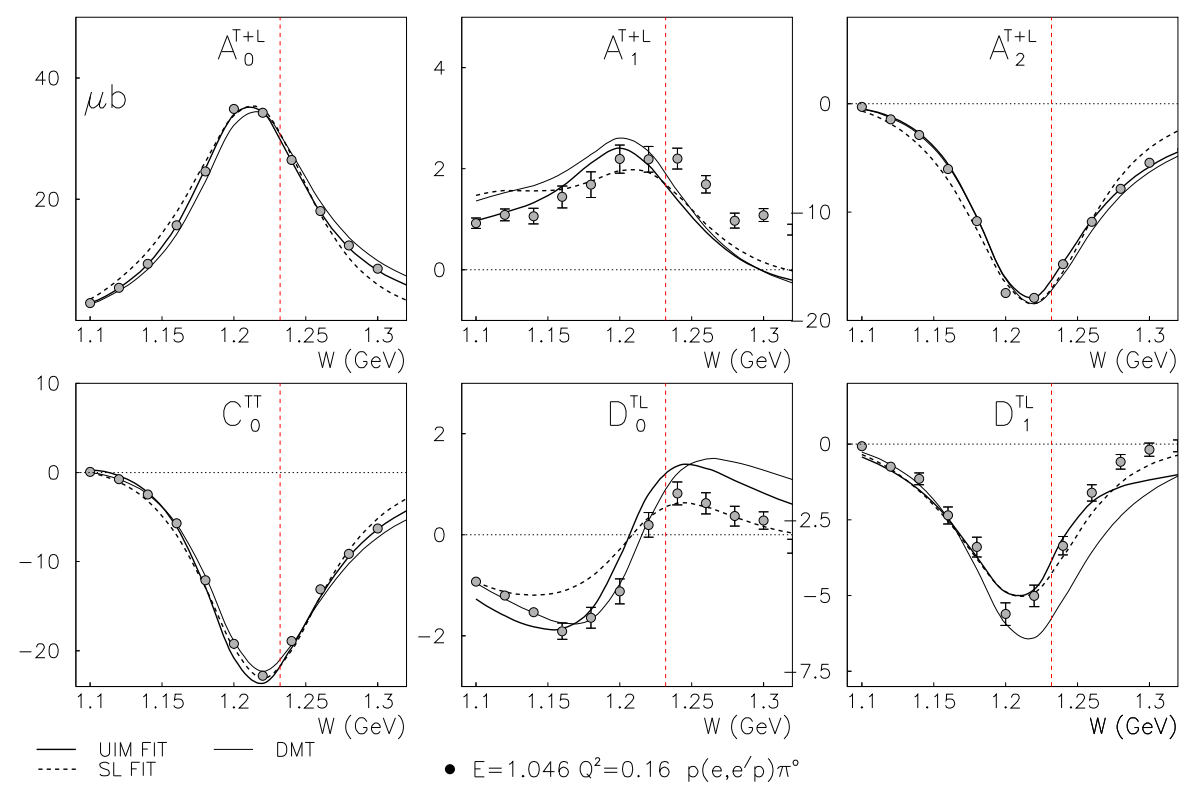

Figure 2. $W$ dependence of partial wave coefficients from Legendre fit of experimental $\pi^{0}$ electroproduction structure functions $\sigma_{T+L}, \sigma_{T T}$ and $\sigma_{T L}$. Solid curve shows UIM fit results. Vertical line shows position of $\Delta(1232)$ resonance. CLAS data 33. taken at $\mathrm{E}=1.046 \mathrm{GeV}$ and $Q^{2}=0.16 \mathrm{GeV}^{2}$.

\subsection{Resonance transition form factors from $\pi, \eta, 2 \pi$ production data}

Pion electroproduction provides new information not available using real photons. First, measuring the $Q^{2}$ dependence of the photocoupling amplitudes probes a range of distance scales within the nucleon, revealing spatial information about the constituents. Second, polarization of the virtual photon allows study of both longitudinal and transverse modes of resonance excitation. Both of these factors place strong constraints on the structure of the transition current used in phenomenological, quark model and lattice QCD calculations.

3.1.1. $\Delta(1232)$ Resonance A longstanding question is the origin of the non-zero quadrupole strength experimentally observed in the $\gamma^{*} p \rightarrow \Delta(1232)$ transition. Quadrupole transitions, which occur via the absorption of $E 2$ or $C 2$ photons, imply non-zero orbital angular momentum in the $N$ or $\Delta$ wave function, which can arise from configuration mixing due to residual gluon or meson interactions between constituent quarks or from the direct photon coupling to the pion cloud. Until recently the data were not sufficiently accurate to constrain calculations. New precision measurements of the $p\left(e, e^{\prime} p\right) \pi^{o}$ reaction at BATES, JLAB and MAMI have drastically improved our knowledge of the $Q^{2}$ dependence of the electric and Coulomb quadrupole/magnetic dipole ratios $R_{E M}=E_{1+} / M_{1+}$ and $R_{S M}=S_{1+} / M_{1+}$. The large acceptance CLAS experiments at JLAB measure the interference terms $\operatorname{Re}\left(E_{1+} M_{1+}^{*}\right)$ and $\operatorname{Re}\left(S_{1+} M_{1+}^{*}\right)$ via the azimuthal and partial wave decomposition of the $p \pi^{0}$ final state:

$$
\frac{d \sigma}{d \Omega_{\pi}^{*}}=\sum_{\ell=0}^{2} A_{\ell}^{T+L} P_{\ell}\left(\cos \theta_{\pi}^{*}\right)+\sin ^{2} \theta_{\pi}^{*} C_{0}^{T T} \cos 2 \phi_{\pi}^{*}
$$




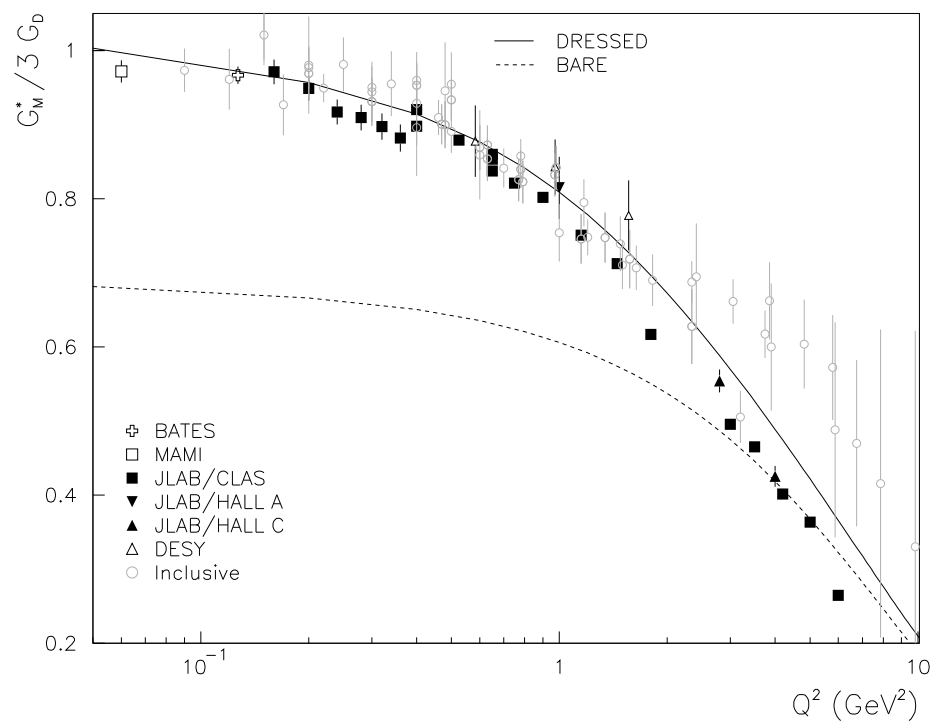

Figure 3. Magnetic dipole transition form factor $G_{M}^{*}$ for $\gamma^{*} N \rightarrow \Delta(1232)$, normalized to the elastic dipole $G_{d}$. Inclusive data are from pre-1990 experiments at Bonn, DESY and SLAC. Other data points were obtained from Unitary Isobar Model analysis of the exclusive $p\left(e, e^{\prime} p\right) \pi^{o}$ reaction. Curves from Sato-Lee model discussed in text.

$$
+\sin \theta_{\pi}^{*} \sum_{\ell=0}^{1} D_{\ell}^{T L} P_{\ell}\left(\cos \theta_{\pi}^{*}\right) \cos \phi_{\pi}^{*}
$$

where to first order, retaining only $M_{1+}$ dominated terms

$$
\begin{aligned}
& \left|M_{1+}\right|^{2}=A_{0}^{T+L} / 2, \\
& \operatorname{Re}\left(E_{1+} M_{1+}^{*}\right)=\left(A_{2}^{T+L}-2 C_{0}^{T T} / 3\right) / 8, \\
& \operatorname{Re}\left(S_{1+} M_{1+}^{*}\right)=D_{1}^{T L} / 6 .
\end{aligned}
$$

Here "T" and "L" refer to the transverse and longitudinal components of the virtual photon polarization. The data are shown in Figure 2 for $Q^{2}=0.2 \mathrm{GeV}^{2}$ where it is seen that all the Legendre coefficients in (25) show a $W$ dependence consistent with interference terms involving the strong resonant $M_{1+}$. At higher $Q^{2}$, where $M_{1+}$ dominance is no longer valid, corrections to (26-28) can be made using the Unitary Isobar Model (UIM) described in Section 2.1 where non-resonant backgrounds and tails from higher resonances are parameterized using (14) and (15). A typical UIM fit is shown in Figure 2 ,

Figure 3 shows the $Q^{2}$ dependence of the magnetic dipole $G_{M}^{*}$, which is related to the isospin $I=3 / 2 \pi N$ multipole $\operatorname{Im}\left(M_{1+}^{3 / 2}\right)$ through the $\Delta$ width and a kinematic factor. These new exclusive measurements of $G_{M}^{*}$ now extend over the range $Q^{2}=0.06-6.0 \mathrm{GeV}^{2}$, and confirm the rapid $Q^{2}$ falloff relative to the elastic dipole seen in previous inclusive measurements, but with much greater sensitivity to the resonant $M_{1}+$ multipole at higher $Q^{2}$. As previously noted, at least part of this rapid falloff may arise from the 'dressing' of the $\gamma^{*} N \rightarrow \Delta$ vertex by rescattering 

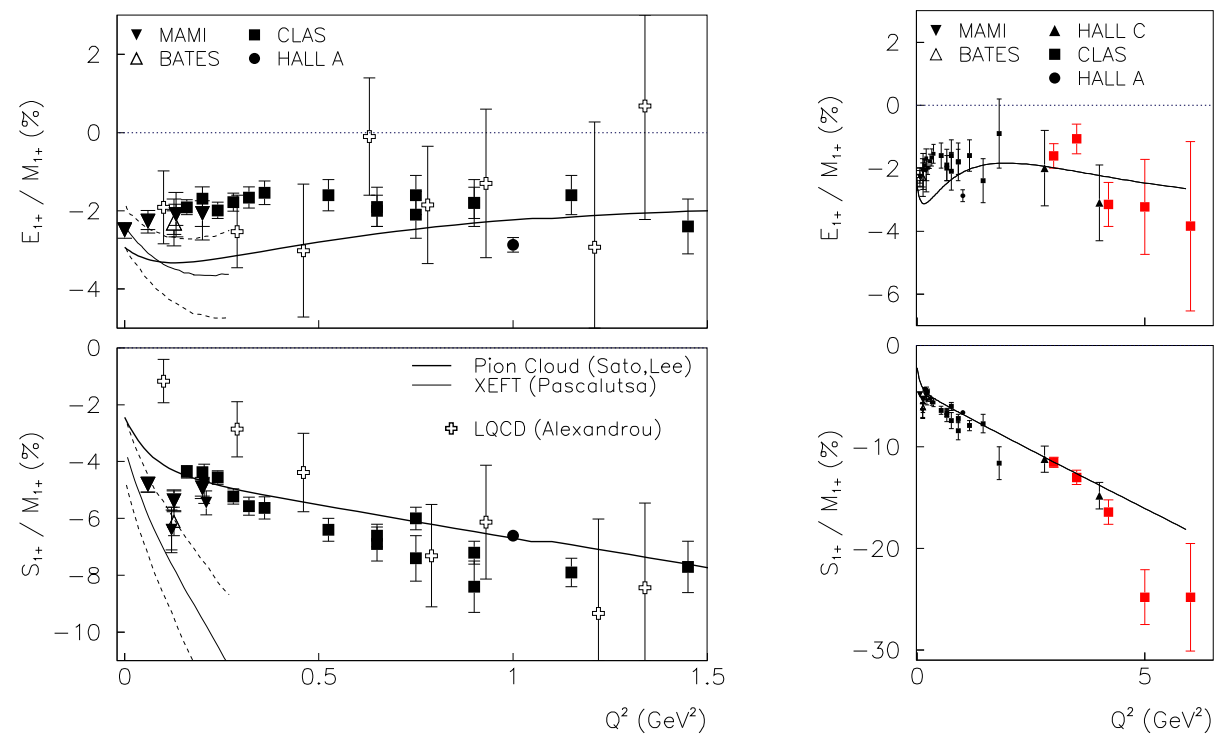

Figure 4. Quadrupole $\gamma N \rightarrow \Delta$ (1232) transition form factors at low $Q^{2}$ (left) and high $Q^{2}$ (right) from recent experiments [31. Electric quadrupole $E_{1+}$ and scalar quadrupole $S_{1+}$ are normalized to the magnetic dipole $M_{1+}$. Preliminary data from CLAS 33, 35 and MAMI 40, 34, are also included.

through the pion cloud. The 'bare' curve in Figure 3 is obtained in the SL dynamical model when the predicted 'dressed' $G_{M}^{*}$ form factor is fitted to the data. It shows the increasing contribution from mesons as $Q^{2} \rightarrow 0$. Similar results have been obtained in the Dubna-Mainz-Taipei model.

The experimental quadrupole transition ratios REM and RSM are shown in Figure 4. The JLAB results are based on a full partial-wave analysis, while the MAMI and BATES data sets cover a more limited angle range. For the latter, MAID, DMT and SL model fits are used to estimate the $\Delta$ resonant multipoles. The other data points with errors are quenched lattice QCD calculations [30] which have undergone a linear chiral extrapolation to the physical pion mass. It is evident that while the calculations qualitatively account for the magnitude of REM and RSM, the $Q^{2}$ dependence is not consistently described. The low $Q^{2}$ underprediction of RSM by LQCD is particularly striking. It was recently demonstrated, using a relativistic chiral effective field theory ( $\chi \mathrm{EFT}$ ) calculation [32, that RSM may be particularly sensitive to a non-analytic dependence of pion loop diagrams on the quark mass, rendering invalid the linear chiral extrapolation used in 30. A strong negative slope near $Q^{2}=0$ is predicted by $\chi$ EFT for both REM and RSM (see Figure 4). While the lowest $Q^{2}$ RSM points from MAMI and BATES appear to agree with this prediction, the data for higher $Q^{2}$ are in better agreement with the SL and LQCD predictions. For REM, the data do not show the strong $Q^{2}$ dependence predicted by $\chi$ EFT near $Q^{2}=0$, and also lie outside of the estimated theoretical uncertainty arising from higher-order effects.

At the largest $Q^{2}$ measured by CLAS (right panel in Figure 4) neither REM nor RSM exhibit the helicity conserving scaling behavior expected in the hard pQCD 

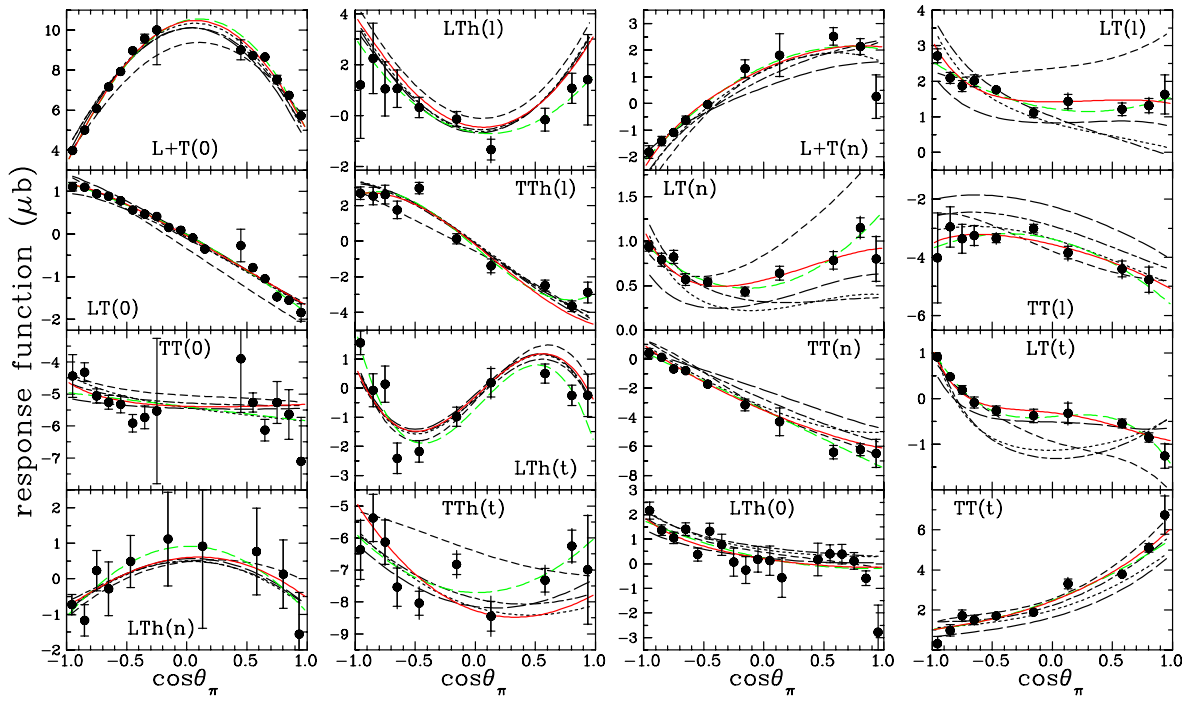

Figure 5. Response functions for $p\left(\vec{e}, e \vec{p}^{\prime}\right) \pi^{0}$ measured by Kelly etal [4, 45] at $W=1.23 \mathrm{GeV}$ and $Q^{2}=1.0 \mathrm{GeV}^{2}$. Notation refers to transverse (t), normal (n) or longitudinal (l) components of the proton recoil polarization. MAID, DMT, SAID and SL models given by dash-dotted, dotted, short-dashed and long-dashed curves.

limit $(\mathrm{REM} \rightarrow 1, \mathrm{RSM} \rightarrow$ constant). Normally the transverse momenta of quarks are expected to be of the order of $\Lambda_{Q C D}$, and thus only important for resonance excitations at low $Q^{2}$. However Idilbi, Ji and Ma 36] have shown that the orbital motion of small $-x$ partons makes a non-neglible contribution to helicity non-conservation in the $N \Delta$ transition, thus invoking both soft and hard QCD processes.

In addition to understanding the long-range chiral dynamics which are evidently important near $Q^{2}=0$, there has long been a fundamental interest in the 'bare' $\gamma N \rightarrow \Delta$ vertex, which is presumably more sensitive to the short-range interquark potential relevant to QCD-motivated models. The bare couplings, which also contain information about the quark core wave functions of the $N$ and $\Delta$, can be estimated in dynamical reaction models by treating them as free parameters to be fitted to the experimental data. This was performed using the SL model for all recent data sets below $Q^{2}=2.0 \mathrm{GeV}^{2}$. A typical fit to CLAS and MAMI data at $Q^{2}=0.2 \mathrm{GeV}^{2}$ is shown in Figure 6] while the $Q^{2}$ dependence of the extracted bare couplings $g_{m}, g_{e}$ and $g_{c}$ are shown in Figure 7 The simplest assumption, that the bare electromagnetic couplings have an identical $Q^{2}$ dependence, is clearly ruled out by the data. Unlike $g_{m}$, both $g_{e}$ and $g_{c}$ show a strong departure from smooth behavior at low $Q^{2}$. This behavior may indicate the presence of additional long-range physics not included in the reaction models. It is certain that more experimental data at the lowest $Q^{2}$ would aid the interpretation of these results.

All reaction models make implicit assumptions about the mechanisms responsible for non-resonant backgrounds. This often leads to substantial model dependence in the extraction of resonance parameters due to ambiguities in relative phases, omitted diagrams, etc. In addition these same models are often used to perform the partial wave analysis of the experimental data, which should in principle be a model independent procedure. Clearly, more complete experiments would help resolve these 

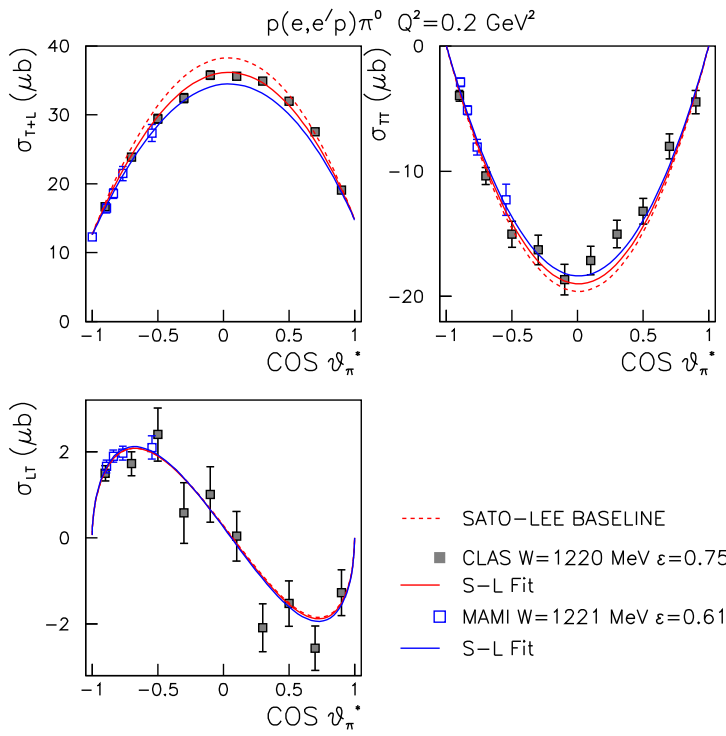

-... SATO-LEE BASELINE

- CLAS W $=1220 \mathrm{MeV} \varepsilon=0.755$

- S-L Fit

MAMI $W=1221 \mathrm{MeV} \varepsilon=0.617$

S-L Fit

Figure 6. Fits of Sato-Lee dynamical model to experimental $p\left(e, e^{\prime} p\right) \pi^{0}$ structure functions $\sigma_{T+L}, \sigma_{T T}$ and $\sigma_{L T}$. Preliminary data from CLAS (solid) 33. and MAMI (open) 34 are shown at $Q^{2}=0.2 \mathrm{GeV}^{2}$. The 'baseline' curve refers to the default $Q^{2}$ parameterization of the 'bare' couplings $g_{m}, g_{e}$ and $g_{c}$ prior to the fit.

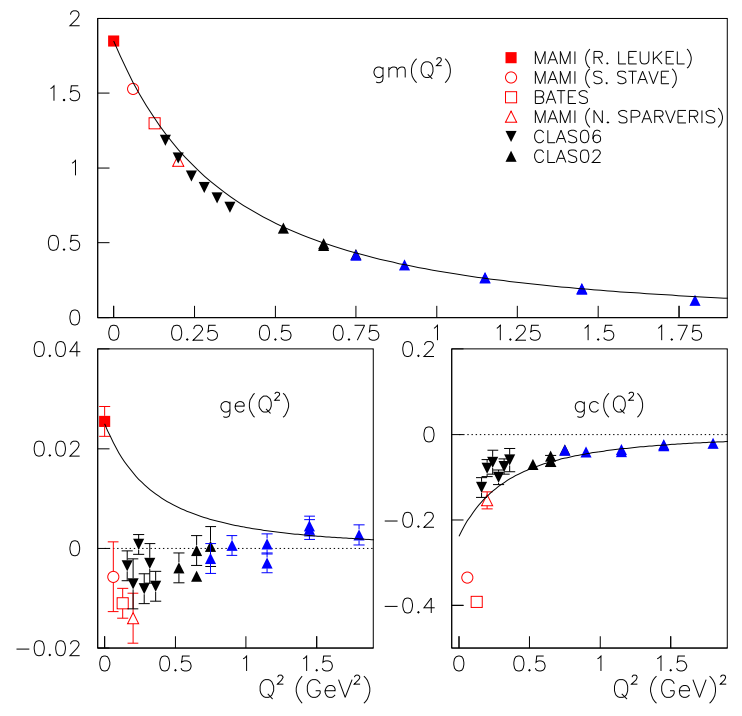

Figure 7. $Q^{2}$ dependence of bare magnetic dipole, electric quadrupole and coulomb quadrupole couplings $g_{m}, g_{e}$ and $g_{c}$ for the $\gamma^{*} p \rightarrow \Delta$ transition, extracted from fits of SL dynamical model to BATES, CLAS and MAMI data. Solid line assumes $Q^{2}$ dependence of $g_{e}$ and $g_{c}$ couplings are identical to $g_{m}$. 
ambiguities. A recent JLAB/Hall A experiment used a polarized beam and a high resolution magnetic spectrometer instrumented with a recoil polarimeter to measure 16 neutral pion electroproduction response functions at $Q^{2}=1.0 \mathrm{GeV}^{2}$. Twelve of these response functions were observed for the first time. These data are shown in Figure 5 at the peak of the $\Delta(1232)$, compared to MAID, DMT, SAID and SL model predictions. Although the overall agreement is good, there are several response functions for which the models clearly diverge in their predictions. This could be due simply to lack of previous experimental information on the weaker underlying multipoles. Due to the phase sensitivity of these polarization observables, a nearly model-independent extraction of $R E M$ and $R S M$ was obtained.

3.1.2. $P_{11}(1440)$ A significant new result using the large acceptance data from CLAS is the demonstration [41] of a large sensitivity to the $M_{1-}^{1 / 2}$ and $S_{1-}^{1 / 2}$ multipoles. These multipoles receive large contributions from excitation of the $I=1 / 2 P_{11}(1440)$ or "Roper" resonance. Due to the isospin coupling, this sensitivity is maximized in the $n \pi^{+}$channel. It turns out that UIM fits to combined data sets which include the helicity dependent asymmetry $A_{L T}^{\prime}\left(n \pi^{+}\right)$and the cross section $d \sigma\left(n \pi^{+}\right)$taken in the second resonance region, significantly constrain the real and imaginary parts of these multipoles through their interference, based on the UIM analysis, with largely Born dominated non-resonant multipoles. The sensitivity of the quantity $\sigma_{L T}^{\prime} \approx d \sigma \cdot A_{L T}^{\prime}$ to the imaginary (resonant) part of these multipoles is demonstrated in the left panel of Figure 8. The plot shows the sensitivity of this observable at $Q^{2}=0.4 \mathrm{GeV}^{2}$ to a $0.5 \mu b^{1 / 2}$ shift in the transverse and longitudinal resonant photocouplings. The sensitivity is the result of the interference term that mixes real and imaginary amplitudes

$$
\sigma_{L T^{\prime}} \sim \operatorname{Im}(L) \cdot \operatorname{Re}(T)-\operatorname{Im}(T) \cdot \operatorname{Re}(L),
$$

where $\mathrm{L}$ and $\mathrm{T}$ represent the longitudinal and transverse amplitudes, respectively. At much higher $Q^{2}$, the tail from the $\Delta(1232)$ is strongly diminished, and the Roper is directly visible as a small bump in the total $n \pi^{+}$cross section near $W=1.4 \mathrm{GeV}$ (right panel).

Using UIM and MAID model fits to CLAS data, the Roper photocoupling amplitudes have now been extracted over a large range of $Q^{2}$, as shown in Figure9, A few of the $Q^{2}$ points have been further analyzed in a multi-channel global fit [42] which includes the $p \pi^{0}$ and $p \pi^{+} \pi^{-}$final states and the agreement with the single channel analysis is good. We also show recently published [43, 45] points at $Q^{2}=1.0 \mathrm{GeV}^{2}$ from Hall A. The new CLAS06 data at even higher $Q^{2}$ [46] confirm the zero-crossing of $A_{1 / 2}^{p}$ first reported in [42. This feature can completely rule out some previous quark model calculations, such as most non-relativistic approaches, as well as the quarkgluon hybrid approach for which no zero crossing occurs and for which $S_{1 / 2}^{p}=0$. The strong longitudinal response seen in these data could revive the traditional 'breathing mode' description of the Roper, possibly augmented by coupling to the $q \bar{q}$ cloud of the nucleon as suggested by the good agreement with the VMD model of Cano and Gonzales [49. Dynamical models and lattice QCD calculations could provide useful input towards intepreting the zero-crossing seen in $A_{1 / 2}^{p}$.

3.1.3. Second and Third Resonance Regions The most prominent states for $W>$ $1.5 \mathrm{GeV}$ are $S_{11}(1535), D_{13}(1520)$, and $F_{15}(1680)$. These have fairly unambiguous 

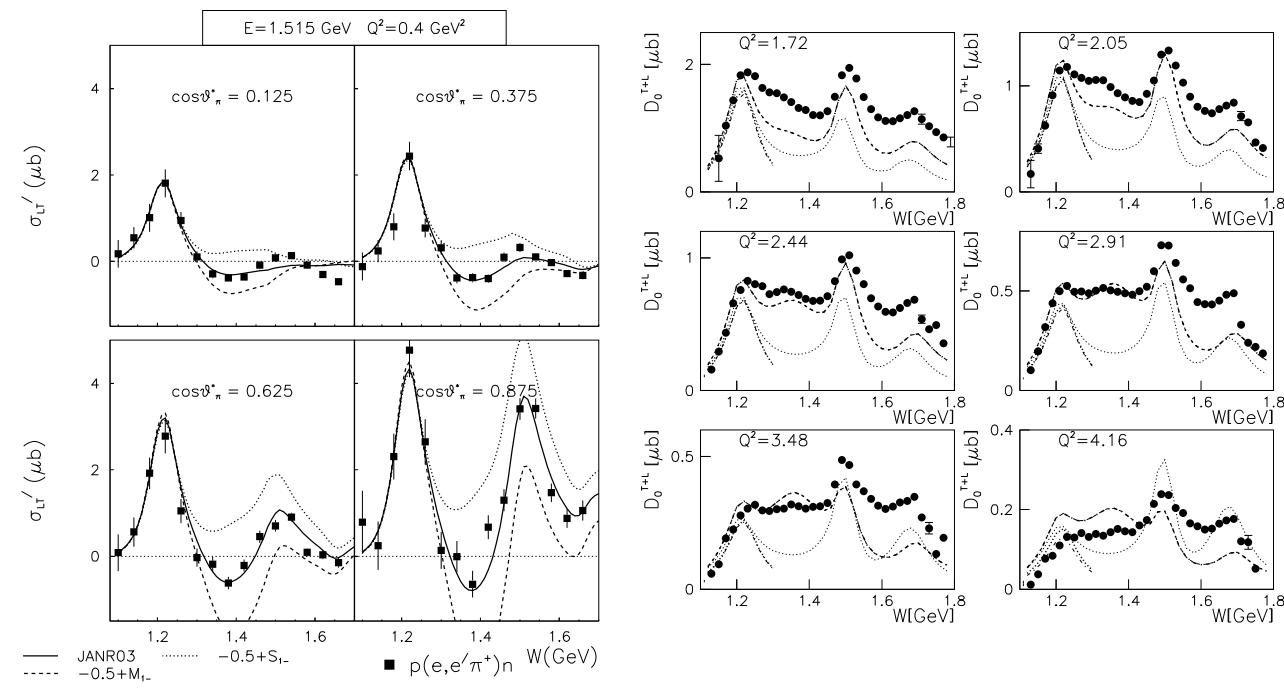

Figure 8. CLAS measurement of the $W$ dependence of $\sigma_{L T^{\prime}}$ (left) and $\sigma_{T+L}$ (right) for the $p\left(e, e^{\prime} \pi^{+}\right) n$ reaction. The curves show UIM (left) and MAID (right) model sensitivity to changes in multipoles $M_{1-}^{1 / 2}$ and $S_{1-}^{1 / 2}$ involved in formation of the Roper resonance. Data were taken at $Q^{2}=0.4 \mathrm{GeV}^{2}$ (left) and $Q^{2}=1.72-4.16 \mathrm{GeV}^{2}$ (right).
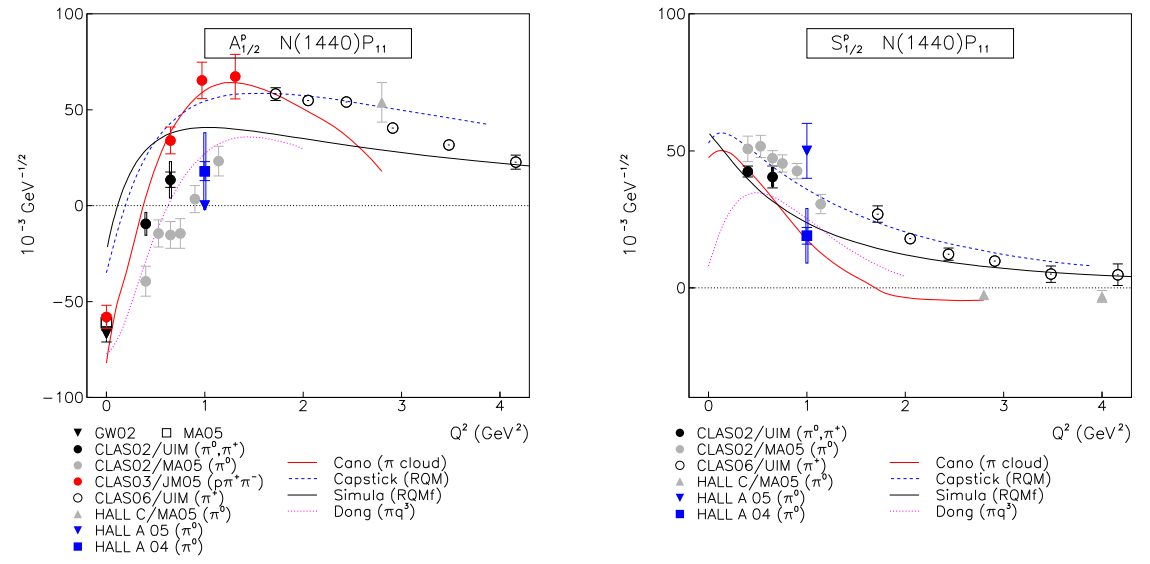

Figure 9. $Q^{2}$ evolution of the $P_{11}(1440)$ (Roper) amplitudes $A_{1 / 2}^{p}$ and $S_{1 / 2}^{p}$. Both CLAS/UIM and Hall A points include model error. The MA05 analyses 56 are dominated by $p \pi^{0}$ data. Other points are GW02 [70]. The curves are model calculations [49, 53, 55, 52, 54,50, described in the text. 

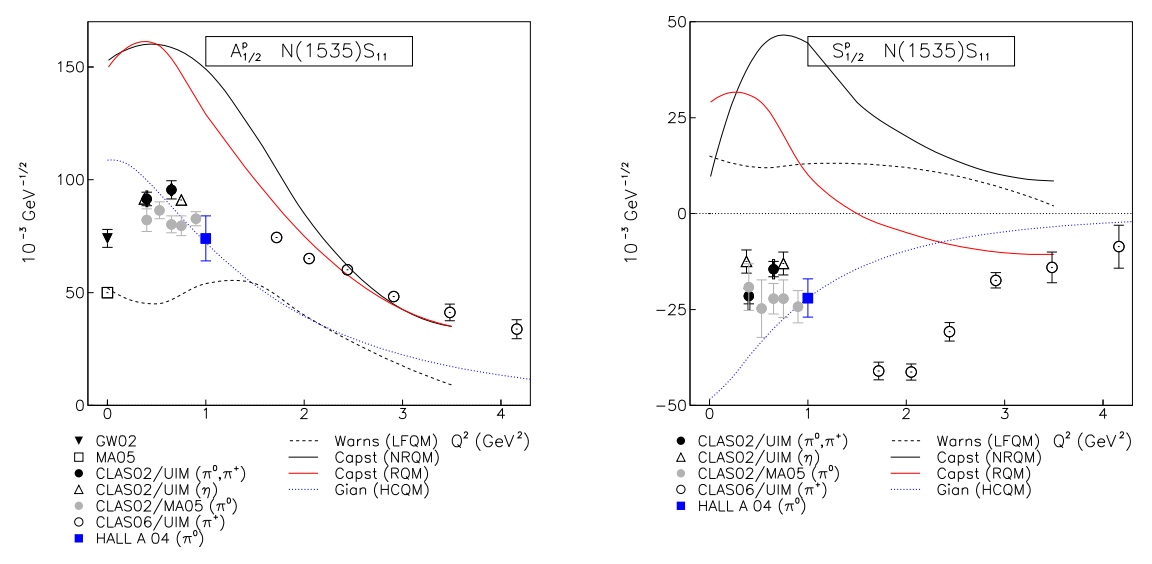

Figure 10. $Q^{2}$ evolution of the $\gamma p \rightarrow S_{11}$ (1535) photocouplings amplitudes $A_{1 / 2}$ and $S_{1 / 2}$. See Figure 9 for explantion of notation.

signatures in partial wave analyses and can be reasonably well isolated in single pion production channels. Furthermore, except for the $S_{11}$, the $\pi N$ branching fractions and widths are known sufficiently to extract photocouplings with $\approx 10 \%$ accuracy. On the other hand, a consistent quark model description of the $Q^{2}$ dependence of these three orbital excitations has proven elusive. The new preliminary CLAS $p\left(e, e^{\prime} \pi^{+}\right) n$ data (labeled CLAS06 in Figures 9[12) has extended the study of $N \rightarrow N^{*}$ transition form factors into the unexplored region above $Q^{2}=2 \mathrm{GeV}^{2}$.

For the $S_{11}(1535)$, the CLAS/UIM determination 42 of photocoupling amplitudes from $\pi$ and $\eta$ electroproduction channels (assuming branching fractions $\beta_{\pi N}=0.4, \beta_{\eta N}=0.55$ and $\Gamma_{t o t}=150 \mathrm{MeV}$ ) give results which are in good agreement up to the highest $Q^{2}$ measured, unlike at $Q^{2}=0$ where $A_{1 / 2}^{p}$ from $\pi$ production is smaller than for $\eta$ production (see Figure 10). It has been shown 47, 48 that strong channel-coupling effects complicate the analyses near $Q^{2}=0$, although the $Q^{2}$ evolution of these effects has not been studied. The new data at higher $Q^{2}$ show a more dipole-like form factor compared to the anomalously hard form factor seen for $Q^{2}<2 \mathrm{GeV}^{2}$. Also for the first time a reliable measurement of the longitudinal photocoupling has been made, which may shed more light on the role of meson rescattering.

A more precise determination of the $\gamma^{*} p \rightarrow D_{13}(1520)$ and $\gamma^{*} p \rightarrow F_{15}(1680)$ transitions has resulted from the increased $I=1 / 2$ sensitivity provided by the CLAS $n \pi^{+}$data. The helicity non-conserving transverse amplitude $A_{3 / 2}^{p}$ for both transitions is clearly dominant at $Q^{2}=0$ (see Figs. 11 and 12), and falls very rapidly with increasing $Q^{2}$. All quark models predict that at high $Q^{2}$, the helicity conserving $A_{1 / 2}^{p}$ will dominate the transition. However neither the harmonic oscillator potential nor hypercentral potential calculations shown in the figures describe the $Q^{2}$ dependence satisfactorily. This is especially true for the longitudinal couplings (see also Figure 13), where the models fail to consistently predict even the sign. For example, quark model wavefunctions obeying $S U(6) \otimes O(3)$ symmetry predict $S_{1 / 2}^{p}\left(S_{11}\right) \approx-S_{1 / 2}^{p}\left(D_{13}\right)$ [51, in disagreement with the data.

Most higher lying states $(M>1.65 \mathrm{GeV})$ have large $2 \pi$ branching fractions and 

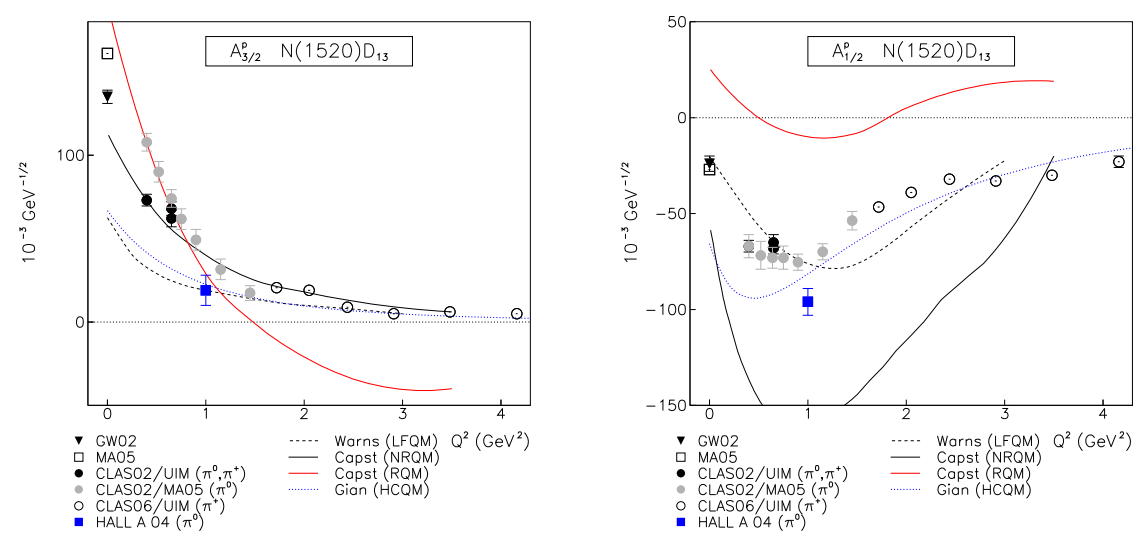

Figure 11. $Q^{2}$ evolution of the $\gamma p \rightarrow D_{13}(1520)$ transverse photocoupling amplitudes $A_{1 / 2}$ and $A_{3 / 2}$. See Figure 9 for explantion of notation.
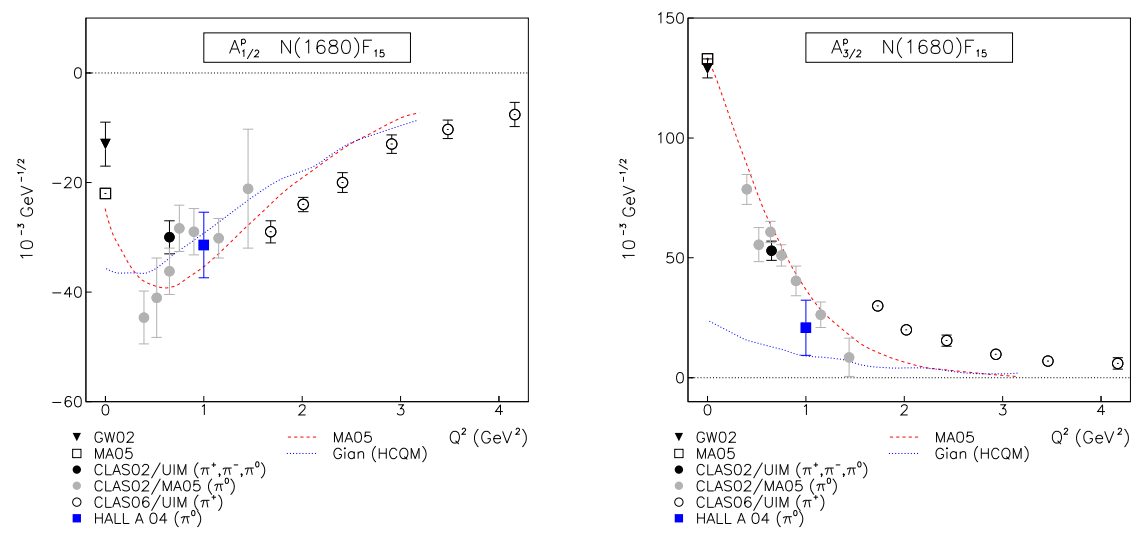

Figure 12. $Q^{2}$ evolution of the $\gamma p \rightarrow F_{15}(1680)$ transverse photocoupling amplitudes $A_{1 / 2}$ and $A_{3 / 2}$. See Figure 9 for explantion of notation.

reliable photocoupling amplitudes can only be obtained by performing (at minimum) fits to measurements of both single and double pion production channels. Also, detection of two pions permits the study of possible 'missing' resonances which have weak couplings to single meson channels. These broad, overlaping states in the mass region $W>1.8 \mathrm{GeV}$ are predicted to decay to both $\Delta \pi$ and $N \rho$, which makes the $\pi^{+} \pi^{-}$channel ideal for their detection. However, the reliable extraction of $N^{*}$ photocouplings is also hampered by a poor understanding of the dynamical mechanisms behind $2 \pi$ production. Currently the most accurate data exists in the second resonance region, where it may be easier to disentangle the numerous resonant and non-resonant subprocesses which contribute to the $2 \pi$ channel.

The analyses [59, 60, 61 of two pion production by using the JLab-Moscow State University (JLAB-MSU) isobar model considered only the minimum set of the tree 

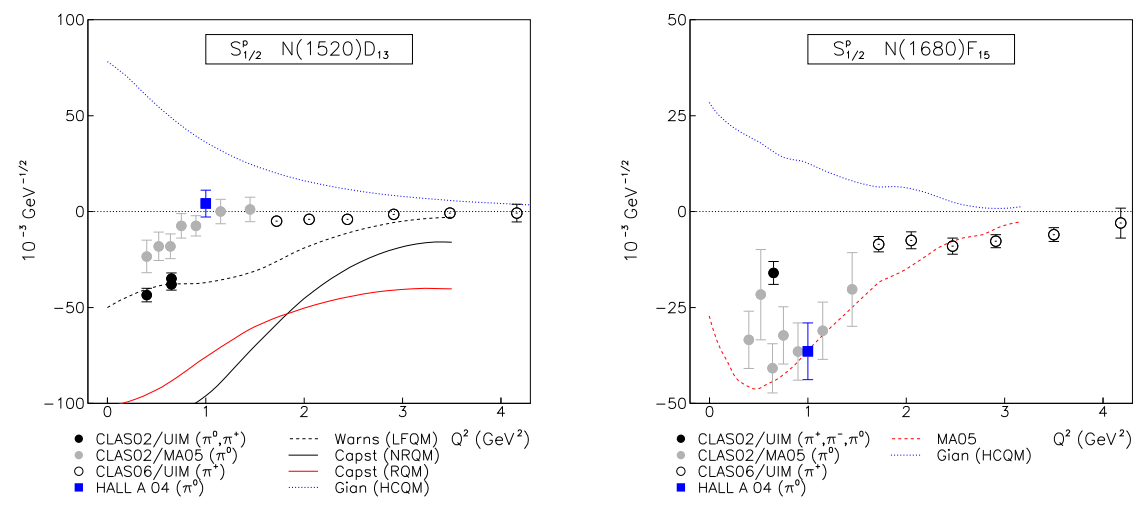

Figure 13. $Q^{2}$ evolution of the longitudinal photocoupling amplitudes $S_{1 / 2}$ for the $\gamma p \rightarrow D_{13}(1520)$ and $\gamma p \rightarrow F_{15}(1680)$ transitions. See Figure 9 for explantion of notation.
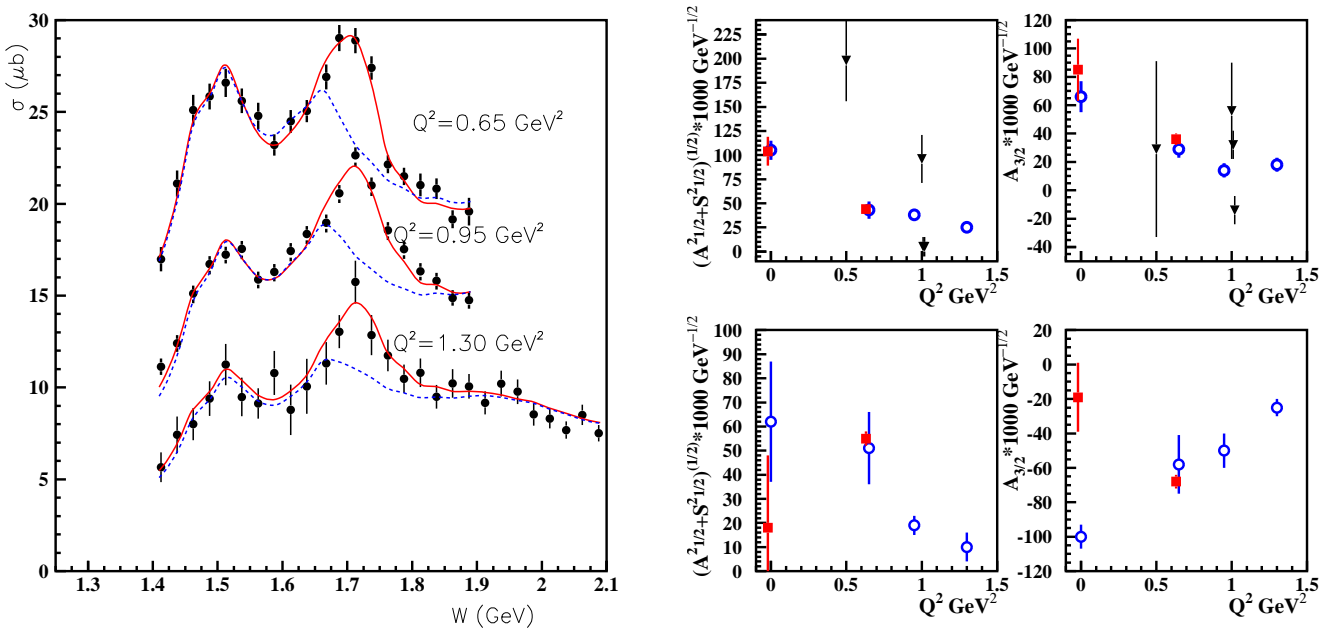

Figure 14. Left: Total $p \pi^{+} \pi^{-}$electroproduction cross sections from CLAS [59]. Solid line: Fit using the JM05 isobar model [57]. Dashed line: JM05 with new $3 / 2^{+}(1720)$ candidate state taken out. Right: $Q^{2}$ dependence of photocouplings for the $D_{33}(1700)$ (top) and $P_{13}(1720)$ (bottom) states. Open circles are results extracted from CLAS $2 \pi$ data 57. Other points are previous $1 \pi$ analyses.

diagrams proposed in the original work of Lüke and Söding 62. However, they made two improvements. They included all 3-star and 4-star resonances listed by the Particle Data Group and used the absorptive model to account for the initial and final state interactions. They found that the $\pi N \Delta$ form factor is needed to get agreement with the data of $\gamma p \rightarrow \pi^{-} \Delta^{++}$, while the initial and final state interactions are not so large. 
In analyzing the two-pion electro-production data, they further included a $\pi \pi N$ phasespace term with its magnitude adjusted to fit the data. This term was later replaced by a phenomenological particle-exchange amplitude which improves significantly the fits to the data. Evidence for intermediate isobar channels such as $\pi^{+} D_{13}(1520)$ and possibly $\pi^{+} F_{15}(1685)$ and $\pi^{-} P_{33}(1600)$ are suggested by these fits. Most prominently, for increasing $Q^{2}$, the $2 \pi$ data show a peak emerging at $W \approx 1.7 \mathrm{GeV}$ (Figure 14) which is identified [61] as a $N^{*}\left(\frac{3}{2}^{+}, 1720\right)$ state. However, this state differs from the conventional $P_{13}(1720)$, having a much stronger photocoupling strength and larger $\pi \Delta / \rho N$ decay ratio compared to PDG. The answer to whether this is a new state or a better experimental determination of an existing resonance awaits further analysis.

Polarization can play an important role in the identification of specific subprocesses which contribute to $2 \pi$ production. A good example is beam-helicityasymmetry data in the $\vec{\gamma} p \rightarrow p \pi^{+} \pi^{-}$reaction measured by Strauch et al. [58] using CLAS with a circularly polarized photon beam in the mass range $W=1.35-2.30 \mathrm{GeV}$. The asymmetry displayed in Figs. 15/16] is defined as

$$
I^{\odot}=\frac{1}{P_{\gamma}} \cdot \frac{\sigma^{+}-\sigma^{-}}{\sigma^{+}+\sigma^{-}},
$$

where $P_{\gamma}$ is the circular polarization of the photon and $\sigma^{ \pm}$are the cross sections for the photon helicity states $\lambda_{\gamma}= \pm 1$. Figure 16] shows a Fourier analysis of the asymmetries measured in the mass region of the $N(1520)$, compared to model calculations incorporating $\pi \Delta$ and $\rho N$ quasi-two-body intermediate states. The $\Delta(1232)$ bump seen in the $M\left(p \pi^{-}\right)$invariant mass (Figure16) for the $a_{1}$ coefficient is suggestive of the sequential decay $N(1520) \rightarrow \pi^{+} \Delta^{0} \rightarrow \pi^{+} \pi^{-} p$. Previous unpolarized cross section measurements showed strong $\Delta$ signatures only in the $M\left(p \pi^{+}\right)$distributions, from the isospin favored $D_{13} \rightarrow \pi^{-} \Delta^{++}$transition. This again illustrates the sensitivity of polarization to the relative phases between various amplitudes, which can selectively amplify combinations of interfering background and resonance amplitudes.
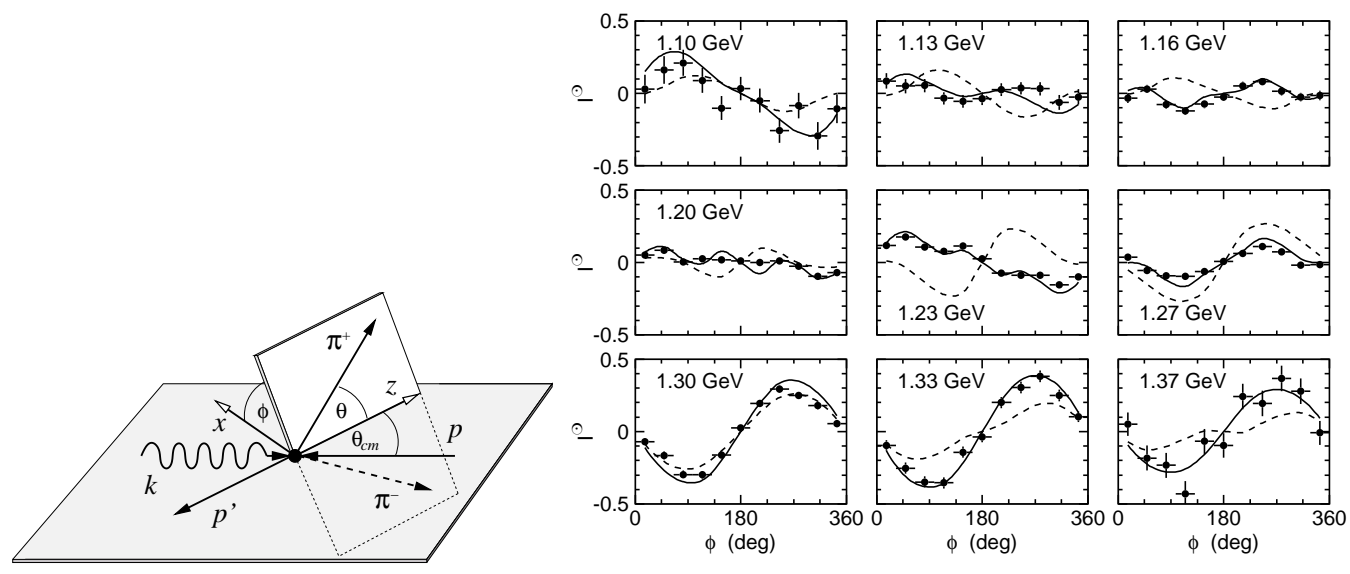

Figure 15. Helicity asymmetries 58 integrated over CLAS acceptance at $W=1.50 \mathrm{GeV}$. Each panel indicates the value of the invariant mass $M\left(p \pi^{+}\right)$. The solid, dashed curves are calculations by Mokeev et al.60, 61 and Fix and Arenhövel 63, respectively. Inset shows the definitions of the azimuthal angle $\phi$ for the reaction $\vec{\gamma} p \rightarrow p \pi^{+} \pi^{-}$. 

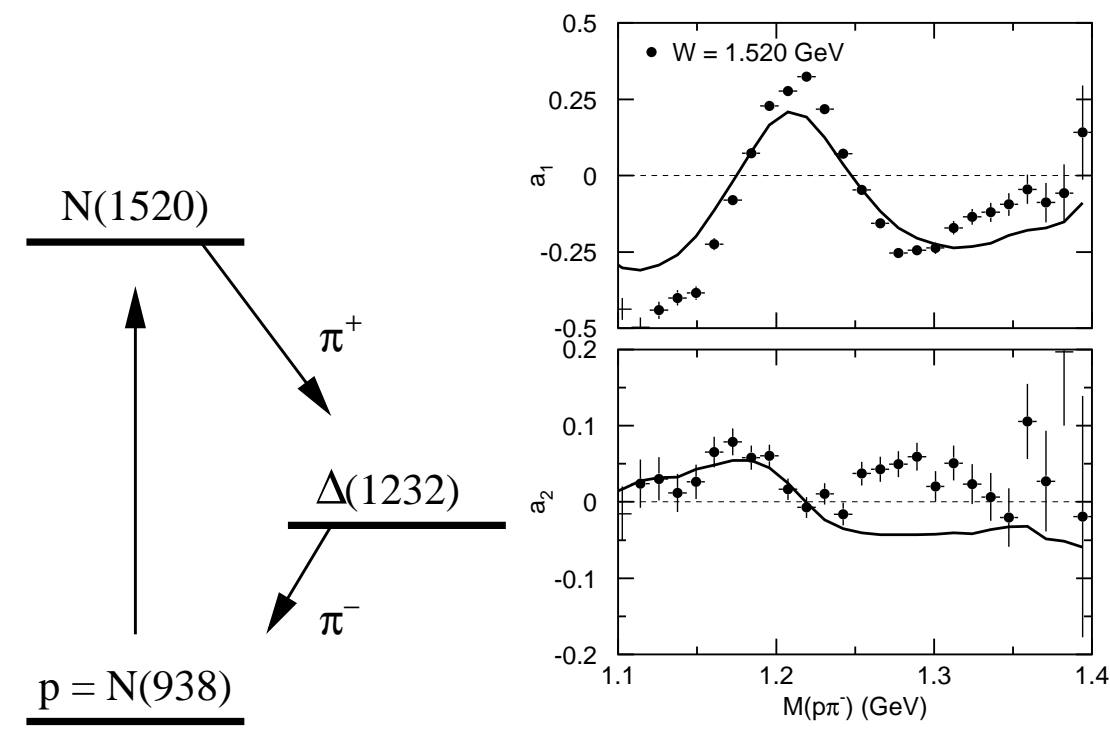

Figure 16. Fourier coefficients $a_{1}$ and $a_{2}$ of the helicity asymmetry as a function of the invariant mass $M\left(p \pi^{-}\right)$for $W=1.520 \mathrm{GeV}$. The curves are the results of Fix and Arenhövel 63. The diagram illustrates the sequential decay $N(1520) \rightarrow \pi^{+} \Delta^{0} \rightarrow \pi^{+} \pi^{-} p$ which might be observed in the data.

\subsection{Analyses of $\omega N, K \Lambda, K \Sigma$ production data}

Because the production cross sections of the $\omega N, K \Lambda, K \Sigma$ channels are much weaker than those of the $\pi N$ and $2 \pi N$ channels, tree-diagram models cannot be used reliably to extract the $N^{*}$ information from the data of photo- and electro-production of these weak channels. We therefore review only the results from coupled-channel analyses.

Most extensive analyses [14, 15, 16, of $K \Lambda, K \Sigma$ and $\omega N$ photoproduction data have been performed by using the K-matrix coupled-channel models. The dynamical coupled-channel model has only been applied to analyze 20, 67 the $K \Lambda$ photoproduction. The main focus of these analyses is to identify possible new resonances and also to extract more accurate information on $N^{*}$ parameters.

The $\omega N$ photoproduction has been analyzed by the Giessen group with the Kmatrix coupled-channel model, defined by (21), with $\gamma N, \pi N, \eta N, 2 \pi N, \omega N$ channels. The $2 \pi N$ channel is approximated as a stable particle channel with its coupling to the $\pi N$ channel constrained by the empirical partial-wave amplitudes [69] of $\pi N \rightarrow \pi \pi N$. The interaction $V$ of (21) is defined by a set of phenomenological Lagrangians with the parameters and associated form factors adjusted to fit the empirical amplitudes of $\gamma N \rightarrow \pi N$, the cross sections of $\pi N, \gamma N \rightarrow \eta N$ and $\pi N, \gamma N \rightarrow \omega N$.

In Figure 17, we show their fits to the $\gamma N \rightarrow \omega N$ differential cross sections. They found a strong contribution from the $D_{15}(1675)$ resonance to $\pi N \rightarrow \omega N$ and from the $F_{15}(1680)$ resonance to $\gamma N \rightarrow \omega N$. From their analysis in Ref [14, they also extracted the $\gamma N \rightarrow N^{*}$ helicity amplitudes. Their results are listed in the first rows of Tables 12. The SAID values from the VPI-GWU single-channel analysis are shown in the second rows. The differences between these two analysis results indicate the importance of including the coupled-channel effects in extracting the $N^{*}$ parameters.

Experimental results for $K \Lambda$ and $K \Sigma$ production have become very extensive 


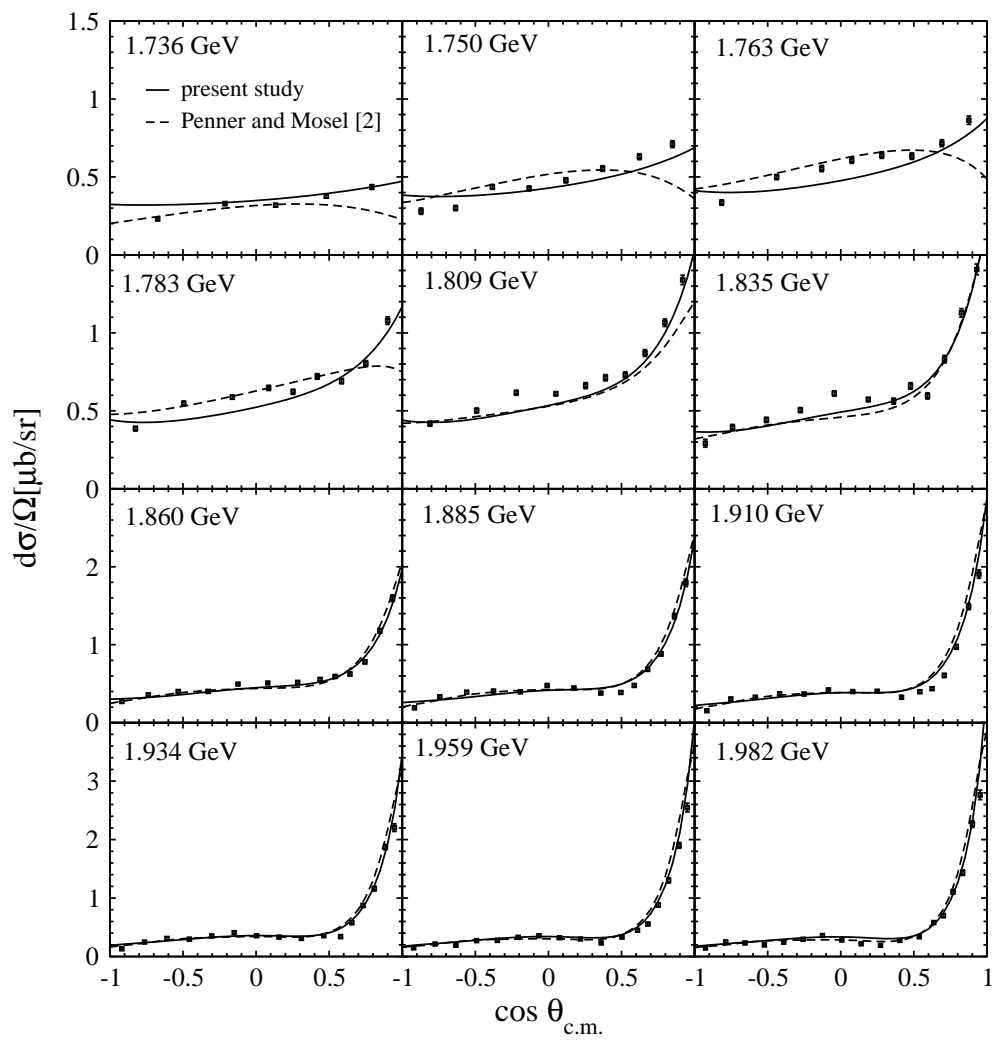

Figure 17. Fits of the coupled-channel model calculations of the Giessen group [12] to experimental $\gamma N \rightarrow \omega N$ differential cross sections from SAPHIR [66].

over the past decade. However the coupled-channel analyses of these data are only now becoming available. The Giessen group, by extending their model to include $K Y$ channel, have found that $S_{11}(1650), P_{13}(1720)$ and $P_{13}(1900)$ are the main resonance contributions to $K \Lambda$ photoproduction. They show that a peak near $1.9 \mathrm{GeV}$, which was identified with the "missing" $D_{13}(1960)$ in a tree-diagram model analysis [71, can be described by a coherent sum of the contributions from the "known" resonant and non-resonant amplitudes. This clearly demonstrates the importance of using a coupled-channel approach to analyze the data of any channel, such as the $K Y$ and $\omega N$ channels, having much smaller production cross sections than the $\pi N$ and $2 \pi N$ channels.

The KVI group has analyzed the $K \Lambda$ and $K \Sigma$ photoproduction data using a Kmatrix coupled-channel model with $\gamma N, \pi N, \eta N, K \Lambda, K \Sigma$, and $\phi N$ channels. They use the $\mathrm{SU}(3)$ Lagrangian to define the interaction $\mathrm{V}$ of (21). With appropriate choices of form factors, they found that the data can be described very well with most of the coupling parameters not too different from their SU(3) limit. Their fits to the data on $K \Lambda$ and $K \Sigma$ total cross sections are shown in Figure 18. They found very large contributions from both resonances and coupled-channel effects.

The $K \Lambda$ photoproduction has also been analyzed by using a dynamical coupled- 
Table 1. Helicity amplitudes of $I=\frac{1}{2}$ resonances (in $10^{-3} \mathrm{GeV}^{-1 / 2}$ ) determined in the Giessen study (first line); second line: results of SAID group [70; "n.g.": not given.

\begin{tabular}{l|ll|ll}
\hline$L_{2 I, 2 S}$ & $A_{1 / 2}^{p}$ & $A_{1 / 2}^{n}$ & $A_{3 / 2}^{p}$ & \multicolumn{1}{c}{$A_{3 / 2}^{n}$} \\
\hline$S_{11}(1535)$ & 92 & -13 & \multicolumn{2}{|c}{-} \\
& $30(3)$ & $-16(5)$ & \multicolumn{2}{|c}{-} \\
$S_{11}(1650)$ & 57 & -25 & \multicolumn{2}{|c}{-} \\
& $74(1)$ & $-28(4)$ & \multicolumn{2}{|c}{-} \\
\hline$P_{11}(1440)$ & -84 & 138 & \multicolumn{2}{|c}{-} \\
& $-67(2)$ & $47(5)$ & \multicolumn{2}{|c}{-} \\
$P_{11}(1710)$ & -50 & 68 & & - \\
& $7(15)$ & $-2(15)$ & \multicolumn{2}{|c}{-} \\
\hline$P_{13}(1720)$ & -65 & 1 & 35 & -4 \\
& $-15(15)$ & $7(15)$ & $7(10)$ & $-5(25)$ \\
$P_{13}(1900)$ & -8 & -19 & 0 & 6 \\
& n.g. & & & -141 \\
\hline$D_{13}(1520)$ & -13 & -70 & 145 & $-112(3)$ \\
& $-24(2)$ & $-67(4)$ & $135(2)$ & -33 \\
$D_{13}(1950)$ & 11 & 40 & 26 & \\
& n.g. & & & -84 \\
\hline$D_{15}(1675)$ & 9 & -56 & 21 & $-71(5)$ \\
\hline$F_{15}(1680)$ & $33(4)$ & $-50(4)$ & $9(3)$ & -48 \\
& $-13(2)$ & $29(6)$ & 116 & $-58(2)$ \\
$F_{15}(2000)$ & 11 & 9 & 25 & -3 \\
& n.g. & & & \\
\hline & & & & \\
\hline
\end{tabular}

Table 2. Extracted helicity amplitudes of $I=\frac{3}{2}$ resonances ( in $10^{-3} \mathrm{GeV}^{-1 / 2}$ ) considered in the Giessen study. Second line: results of SAID group 70]; "n.g.": not given.

\begin{tabular}{l|l|l}
\hline$L_{2 I, 2 S}$ & $A_{1 / 2}^{p}$ & $A_{3 / 2}^{p}$ \\
\hline$S_{31}(1620)$ & 47 & - \\
& $-13(3)$ & - \\
\hline$P_{31}(1750)$ & 34 & - \\
& n.g. & n.g. \\
\hline$P_{33}(1232)$ & -128 & -253 \\
& $-129(1)$ & $-243(1)$ \\
$P_{33}(1600)$ & -13 & -28 \\
& $-18(15)$ & $-25(15)$ \\
$P_{33}(1920)$ & 2 & 8 \\
& n.g. & n.g. \\
\hline$D_{33}(1700)$ & 107 & 147 \\
& $89(10)$ & $92(7)$ \\
\hline$F_{35}(1905)$ & 32 & -89 \\
& $2(5)$ & $-56(5)$ \\
\hline$D_{35}(1930)$ & -53 & -23 \\
& $4(6)$ & $-3(6)$ \\
\hline
\end{tabular}

channel model 67] which is based on an extension of the dynamical model of 4] to include the $K \Lambda$ channel. It differs from the K-matrix coupled-channel models of Giessen and KVI groups in accounting for the off-shell scattering dynamics. This analysis finds that the most relevant known resonances in $\gamma p \rightarrow K^{+} \Lambda$ are $S_{11}(1535)$, 


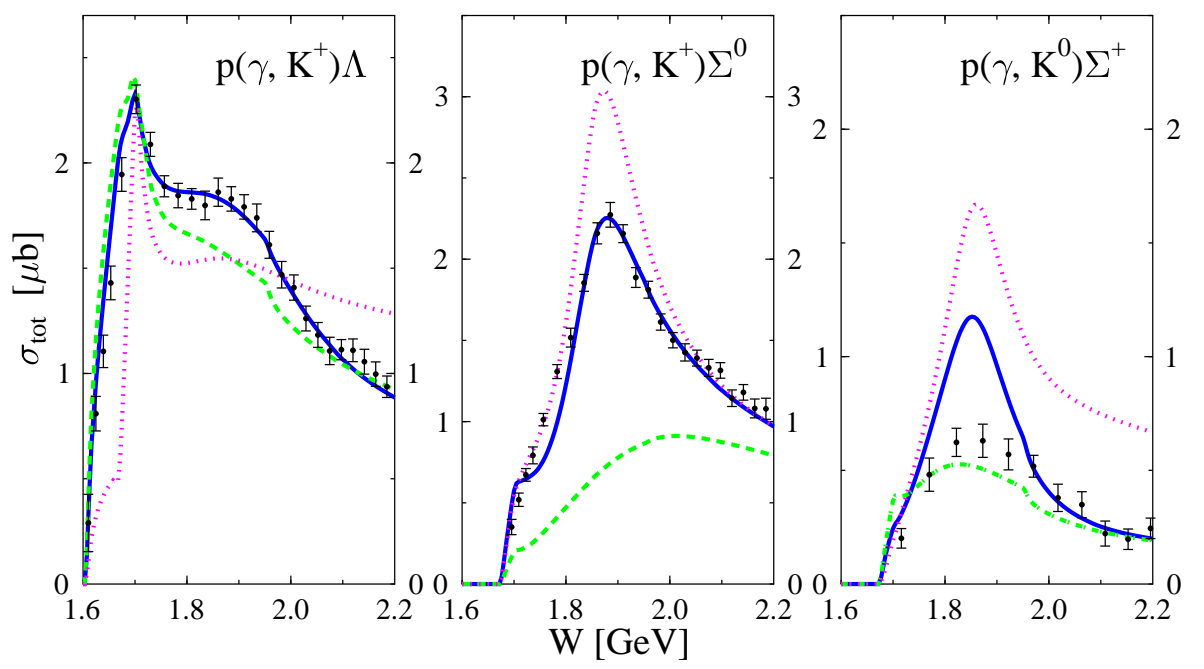

Figure 18. Experimental data on $K \Lambda$ and $K \Sigma$ total cross sections from SAPHIR [64, 65] compared to calculations from KVI group [16]. Dashed line shows effect of turning off all resonance contributions. Dotted line shows coupled rhannel efferts switched off Solid line is full calculation
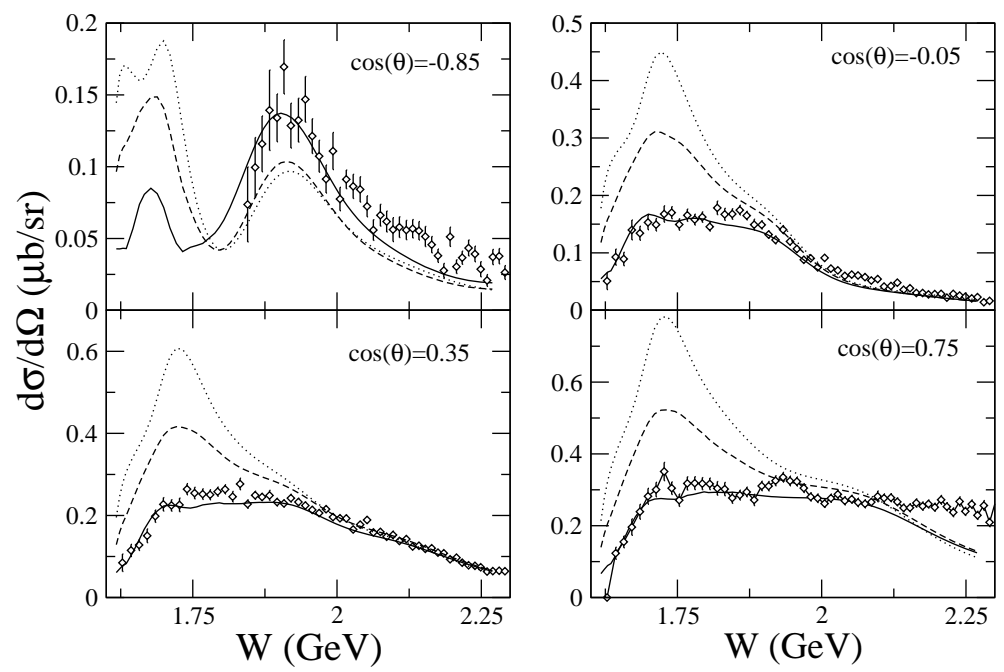

Figure 19. Comparison of the $W$ spectra of $K \Lambda$ production from CLAS 68, at various C.M. angles with model calculations from 67. Solid curves: full calculation. Dotted curves: off-shell scattering turned off. Dashed curves: coupled-channels turned off.

$P_{13}(1900)$ and $D_{13}(1520)$. In addition, they also demonstrate the importance of offshell effects and meson cloud effects in interpreting the extracted $N^{*}$ parameters, as shown in Figure 19. 


\section{Future developments}

In the past few years, we have witnessed very significant progress in the study of $N^{*}$ physics. We now have fairly extensive data for $\pi, \eta, K, \omega, \phi$ and $\pi \pi$ production channels. Many more data will soon be available. Theoretical models for interpreting these new data and/or extracting the $N^{*}$ parameters have also been developed accordingly.

From the analyses of the single pion data in the $\Delta$ region, quantitative information about the $\gamma N \rightarrow \Delta$ transition form factors have been obtained. With the development of dynamical reaction models, the role of pion cloud effects in determining the $\Delta$ excitation has been identified as the source of the long-standing discrepancy between the data and the constituent quark model predictions. Moreover, the $Q^{2}$-dependence of the $\gamma N \rightarrow \Delta$ form factors has also been determined up to about $Q^{2} \sim 6 \mathrm{GeV}^{2}$. The Magnetic $M 1$, Electric $E 2$ and Coulomb $C 2 \gamma^{*} N \rightarrow \Delta$ transition form factors should be considered along with the proton and neutron form factors as benchmark data for testing various hadron models as well as Lattice QCD calculations.

The analyses of the $\pi$ and $\eta$ production data had led to a rather quantitative determination of several $N^{*}$ parameters in the second resonance region. However, a correct interpretation of the $N^{*}$ parameters in terms of current hadron-model predictions requires a rigorous investigation of the dynamical coupled-channel effects which are not included in amplitude analyses based on either the K-matrix isobar model or the dispersion relation approach.

There has significant progress in analyzing the data for $K \Lambda, K \Sigma$, and $\omega N$ photoproduction. It is now generally agreed that these data must be analyzed in a coupled-channel approach. New information on $N^{*}$ parameters have been extracted from K-matrix coupled-channel analyses. The importance of both coupled-channel and meson cloud effects have also been revealed. It will be interesting to extend these analyses to analyze the electroproduction data for also extracting the $N-N^{*}$ transition form factors.

The analysis of $2 \pi N$ production data is still in a development stage, while some interesting results have been obtained from the analyses based on tree-diagram isobar models. A reliable analysis of $2 \pi N$ data must satisfy the $\pi \pi N$ unitarity condition rigorously. Progress in this direction has been made recently. The dynamical coupledchannel model developed in Ref. 28] accounts for all relevant channels and the effects due to the $\pi \pi N$ unitarity condition. It has been applied to reveal important dynamical features due to the $\pi \pi N$ unitary cuts which must be included in extracting the $N^{*}$ information from $2 \pi$ production data. The analyses based on this dynamical coupledchannel approach are being performed at the Excited Baryon Analysis Center (EBAC) at Jefferson Laboratory.

Progress made in the past decade has resulted from close collaborations between experimentalists and theorists. With much more complex data to be analyzed and interpreted, such collaborations must be continued and extended in order to bring the study of $N^{*}$ physics to a complete success.

Acknowledgments This work was supported in part by the U.S. Department of Energy, Office of Nuclear Physics, under Contract No. W-31-109-ENG-38, and in part under Contract No. DE-AC05-84ER40150 and Contract No. DE-AC05-060R23177, 
under which Jefferson Science Associates operates Jefferson Lab.

\section{References}

[1] Capstick S and Roberts W 2000 Prog. Part. Nucl. Phys. 45 S241

[2] Maris P and Roberts C 2003 Int. J. Mod. Phys. E12 297

[3] Arndt R et al 2001 Proc. of the Workshop on the Physics of Excited Nucleons (Mainz) ed D Drechsel and L Tiator (New Jersey: World Scientific) p 467

[4] Sato T and Lee T 1996 Phys. Rev. C54 2660 Sato T and Lee T 2001 Phys. Rev. C63 055201

[5] Kamalov S and Yang S 1999 Phys. Rev. Lett. 834494 Kamalov S, Yang S, Drechsel D, Hanstein O and Tiator L 2001 Phys. Rev. C64 032201(R)

[6] Drechsel D, Hanstein O, Kamalov S and Tiator L 1999 Nucl. Phys. A645 145

[7] Walker R 1969 Phys. Rev. 1821729

[8] Aznauryan I 2003 Phys. Rev. C67 015209

[9] Aznauryan I 2003 Phys. Rev. C68 065204

[10] Aznauryan I 2005 Phys. Rev. C71 015201

[11] Arndt R, Strakovsky I, Workman R 2003 Int. J. Mod. Phys. A18 449

[12] Feuster T and Mosel U 1998 Phys. Rev. C58 457 Feuster T and Mosel U 1999 Phys. Rev. C59 460

[13] Penner G and Mosel U 2002 Phys. Rev. C66 055211 Penner G and Mosel U 2002 Phys. Rev. C66 055212

[14] Shklyar V, Lenske H, Mosel U and Penner G 2005 Phys. Rev. C71 055206

[15] Shklyar V, Lenske H and Mosel U 2005 Phys. Rev. C72 15210

[16] Usov A and Scholten O 2005 Phys. Rev. C72 025205

[17] Cala G, Pascalutsa V, Tjon J and Wright L 2004 Phys. Rev. C70 032201

[18] Yoshimoto T, Sato T, Arima M and Lee T-S 2000 Phys. Rev. C61 065203

[19] Oh Y and Lee T-S 2002 Phys. Rev. C66 045201

[20] Chiang W, Tabakin F, Lee T.-S. and Saghai B 2001 Phys. Lett. B517 101 Julia-Diaz B, Saghai B, Lee T-S and Tabakin F 2006 Phys. Rev. C73 055204

[21] Chen G, Kamalov S, Yang S, Drechsel D and Tiator L 2003 Nucl. Phys. A723 447

[22] Krehl O, Hanhart C, Krewald S and Speth J 2000 Phys. Rev. C62 025270

[23] Fuda M and Alharbi H 2003 Phys. Rev. C68 064002

[24] Gross F and Surya Y 1993 Phys. Rev. C47 703 Surya Y and Gross F 1996 Phys. Rev. C 532422

[25] Kaiser N, Waas T and Weise W 1997 Nucl. Phys. A612 297

[26] Oset E and Ramos A 1998 Nucl. Phys. A635 99

[27] Lutz M and Kolomeitsev E 2002 Nucl. Phys. A700 193

[28] Matsuyama A, Sato T and Lee T-S (in preparation) (2006)

[29] Matsuyama A and Lee T-S 1986 Phys. Rev. C34 1900

[30] Alexandrou C et al 2005 Phys. Rev. Lett. 94021601

[31] Burkert V and Lee T-S 2004 Int. J. of Mod. Phys. E13 1035

[32] Pascalutsa V and Vanderhaeghen M 2006 Phys. Rev. D73 034003

[33] Smith L 2006 Proc. of the Shape of Hadrons Workshop Athens Eds. C.N. Papanicolas and A.M. Bernstein, (AIP), to be published

[34] Sparveris N 2006 Proc. of the Shape of Hadrons Workshop Athens Eds. C.N. Papanicolas and A.M. Bernstein, (AIP), to be published

[35] Ungaro $M$ et al (CLAS Collaboration) 2006 Preprint hep-ex/0606042

[36] Idilbi A, Ji X and Ma J-P 2004 Phys. Rev. D69 014006

[37] Joo K et al (CLAS Collaboration) 2004 Phys. Rev. C70 042201

[38] Joo K et al (CLAS Collaboration) 2003 Phys. Rev. C68 033201

[39] Joo K et al (CLAS Collaboration) 2002 Phys. Rev. Lett. 8812201

[40] Stave S et al 2006 Preprint nucl-ex/0604013

[41] Joo K et al (CLAS Collaboration) 2005 Phys. Rev. C72 058202

[42] Aznauryan I et al 2004 Phys. Rev. C72 045201

[43] Laveissiere G et al (Hall A Collaboration) 2004 Phys. Rev. C69 045203

[44] Kelly J et al (Hall A Collaboration) 2005 Phys. Rev. Lett. 95102001

[45] Kelly J et al (Hall A Collaboration) 2005 Preprint nucl-ex/0509004

[46] Park K et al. (CLAS Collaboration) to be submitted to Phys. Rev. C.

[47] Vrana T, Dytman S and Lee T-S Lee 2001 Phys. Rept. 328181

[48] Arndt R et al. 2005 Phys. Rev. C72 045202 
[49] Cano F and Gonzales P 1998 Phys. Lett. B431 270

[50] Dong Y 1997 J. Phys. G: Nucl. Part. Phys. 231005

[51] Li Z, Dong Y and Ma W 1997 J. Phys. G: Nucl. Part. Phys. 23151

[52] Pace E, Salme G and Simula S 1999 Few Body Syst. Suppl. 10407

[53] Capstick S and Keister B 1995 PR D51 3598

[54] Warns M, Schroder G, Pfiel W and Rollnik H 1990 Z. Phys. C45 627

[55] Aiello M, Giannini M and Santopinto 1998 Eur. J. Phys. G24 753

[56] Tiator L et al. 2004 Eur. J. Phys. A19 55

[57] Mokeev V et al 2005 Preprint hep-ph/0512164

[58] Strauch S et al (CLAS Collaboration) 2005 Phys. Rev. Lett. 95162003

[59] Ripani M et al 2000 Nucl. Phys. A672 220 Ripani M et al 2003 Phys. Rev. Lett. 91022002

[60] Mokeev V et al 2001 Phys. At. Nucl. 641292

[61] Mokkeev V et al 2004 Proc. of the Workshop on the Physics of Excited Nucleons (Grenoble) eds. J Bocquet, V Kuznetsov and D Rebreyend (New Jersey: World Scientific) p 321

[62] Lüke D and Söding P 1971 Springer Tracts in Modern Physics vol 59 (Berlin: Springer)

[63] Fix A and Arenhövel H 2005 Eur. J. Phys. A25 115

[64] Glander K et al 2004 Eur. J. Phys. A19 151

[65] Lawall R et al 2005 Eur. J. Phys. A24 275

[66] Barth J et al 2003 Eur. J. Phys. A18 117

[67] Julia-Diaz B, Saghai B Lee T-S and Tabakin F 2006 Phys. Rev. C73 055204

[68] Bradford R et al (CLAS Collaboration) 2006 Phys. Rev. C73 035202

[69] Manley M 2003 Int. J. of Mod. Phys. A18 441

[70] Arndt R, Strakovsky I and Workman R 1996 Phys. Rev. C53 430 Arndt R et al 1995 Preprint nucl-th/9509005 Arndt R, Briscoe W, Strakovsky I and Workman R 2002 Phys. Rev. C66 055213 Arndt R et al 2002 Preprint nucl-th/0205067

[71] Mart T and Bennhold C 2000 Phys. Rev. C61 12201(R) 